\title{
Energetic Electrons at Uranus: Bimodal Diffusion in a Satellite Limited Radiation Belt
}

\author{
R. S. SELESNICK AND E. C. STONE \\ California Institute of Technology, Pasadena
}

\begin{abstract}
The Voyager 2 cosmic ray experiment observed intense electron fluxes in the middle magnetosphere of Uranus. High counting rates in several of the solid-state detectors precluded the nomal multiple coincidence analysis used for cosmic ray observations, and we have therefore performed laboratory measurements of the single-detector response to electrons. These calibrations allow a deconvolution from the counting rate data of the electron energy spectrum between energies of about 0.7 and $2.5 \mathrm{MeV}$. We present model fits to the differential intensity spectra from observations between $L$ values of 6 and 15. The spectra are well represented by power laws in kinetic energy with spectral indices between 5 and 7 . The phase space density at fixed values of the first two adiabatic invariants generally increases with $L$, indicative of an extemal source. However, there are also local minima associated with the satellites Ariel and Umbriel, indicating either a local source or an effective source due to nonconservation of the first two adiabatic invariants. For electrons which mirror at the highest magnetic latitudes, the local minimum associated with Ariel is radially displaced from the minimum $L$ of that satellite by -0.5 . The latitude variation of the satellite absorption efficiency predicts that if satellite losses are replenished primarily by radial diffusion there should be an increasing pitch angle anisotropy with decreasing $L$. The uniformity in the observed anisotropy outside the absorption regions then suggests that it is maintained by pitch angle diffusion. The effective source due to pitch angle diffusion is insufficient to cause the phase space density minimum associated with Ariel. Model solutions of the simultaneous radial and pitch angle diffusion equation show that the displacement of the high-latitude Ariel signature is also consistent with a larger effective source. This source may be created by inelastic scattering, leading to diffusion in energy as well as pitch angle.
\end{abstract}

\section{INTRODUCTION}

The radiation belts of the outer planets Jupiter, Saturn, Uranus and Neptune have now been explored quite extensively by the Pioneer and Voyager spacecraft. The data have been particularly interesting with regard to the interaction of the trapped radiation with the planetary satellites, which can be a probe of particle transport and loss mechanisms not available at Earth. Uranus and Neptune have the additional feature of a highly tilted dipole magnetic field, and the unusual geometry leads to extended absorption regions outside the satellite orbits. Such a welldefined loss mechanism provides a unique opportunity to study radiation belt diffusion processes.

The first direct measurements of the Uranian radiation belts were made by instrumentation aboard the Voyager 2 spacecraft during its passage through the magnetosphere in January 1986. Both the low-energy charged particle (LECP) and cosmic ray (CRS) experiments measured intense fluxes of trapped electrons, with lower ion fluxes. Preliminary analyses of these results were presented by Krimigis et al. [1986] and Stone et al. [1986]. Detailed analyses of the LECP results, including calculations of energy spectra and pitch angle distributions [Mauk et al., 1987] and phase space densities [Cheng et al., 1987] for both protons and electrons, have subsequently been made. Similar detailed calculations with the CRS data, which would complement the LECP results by their coverage of higher particle energies, have not been made up to now because of uncertainties in the instrumental response, although preliminary calculations were made of the energy spectra [Cooper and Stone, 1986] and the

\section{Copyright 1991 by the American Geophysical Union.}

Paper number 90JA02696.

0148-0227/91/90JA-02696\$05.00 phase space densities [Cooper and Stone, 1991]. In this paper we consider the CRS response to electrons with kinetic energy $\geq 1 \mathrm{MeV}$ based on new calibrations and present results which apply to a region extending from inside the orbit of Ariel to outside the orbit of Umbriel.

Satellite interactions are thought to have produced all of the significant variations in the CRS electron counting rates, which have been described extensively by Cooper and Stone [1991]. The locations of counting rate minima were compared with minimum $L$ values of the satellite orbits based on the $Q 3$ planetary magnetic field model [Connerney et al., 1987; Acuna et al., 1988]. Preliminary analysis of the magnetic field data [Ness et al., 1986] led to an offset tilted dipole (OTD) model of the planetary magnetic field which was largely successful in organizing the particle data, although there were differences between the locations of the counting rate minima and the satellite orbits. Use of the more accurate Q3 model did not eliminate the differences, leading to the realization that the magnetic field configuration alone could not account for the particle profiles. Theoretical calculations [Paonessa and Cheng, 1987; Cooper, 1990] show that the particle absorption rate by a satellite is strongly peaked near the minimum $L$ value, which therefore might be expected to correspond with the minimum particle intensity. However, in several cases the counting rate minima are significantly displaced from the minimum $L$ [Stone et al., 1986; Cooper and Stone, 1991]. Cooper and Stone point out that such offsets may be caused by diffusion of the instantaneous absorption profiles over long time periods.

The offsets in the counting rate minima are an increasing function of energy and are not as apparent at lower energies, either in the counting rates [Krimigis et al., 1986] or in the phase space density analysis of the LECP data [Cheng et al., 1987]. Further analysis of the CRS data is therefore warranted. Our results confirm the preliminary findings of Cooper and Stone 
[1991]. The electron phase space densities do indeed show absorption signatures with offsets from the satellite minimum $L$ values corresponding to those in the counting rate data. There are also deep minima at these locations indicative of a local source of energetic electrons, a result consistent those of Cheng et al.

All of the data analysis refers to $L$ values which were calculated from the Q3 magnetic field model for electrons which mirror at the location of the spacecraft. Drift shell splitting [Stone, 1963] due to the azimuthal asymmetry of the magnetic field is negligible ( $\leq 0.1 \%$ ) outside the Voyager 2 closest approach to Uranus [Acuna et al., 1988]. Also, shell splitting due to large-scale (convection) electric fields is probably negligible because at low energies the counting rate minima are close to the satellite minimum $L$ values [Cooper and Stone, 1991] and the electric field effects decrease with particle energy. The use of $L$ as an invariant drift shell parameter therefore seems justified.

The CRS instrument and the analysis procedure for modeling the electron intensity spectrum are described in section 2 . The resulting model parameters and a subsequent calculation of the electron phase space density, as a function of $L$ and the first two adiabatic invariants of charged particle motion, are presented in section 3. Implications for electron diffusion and model solutions of the bimodal diffusion equation are discussed in section 4.

\section{INSTRUMENT AND DATA ANALYSIS}

The Voyager cosmic ray system includes two high-energy telescopes, four low-energy telescopes and one electron telescope (TET). Each was optimized for sensitivity to the low fluxes of interplanetary cosmic rays. For a complete description see Stone et al. [1977]. The interplanetary data are analyzed by requiring simultaneous observations of a given event in two or more detectors within a given telescope, leading to a unique identification of each analyzed event. The high magnetospheric electron fluxes generally lead to a high rate of accidental coincidences among different detectors, and the multiple coincidence analysis is not possible. The data have therefore been restricted to single detector measurements. For a description of the CRS configuration during the Uranus encounter see Stone et al. [1986] and Cooper and Stone [1991].

We will discuss results from the electron telescope which provided the greatest sensitivity to electrons. A schematic drawing of the eight solid-state detectors within TET is shown in Figure 1. They provide sensitivity to different energy ranges because of the varying amount of passive shielding an electron must penetrate to reach the different detectors. The D1 detector has the least amount of passive shielding due to its location at the front of the telescope. Therefore D1 was most responsive to lower energy electrons and yielded the highest counting rate. The most central detector, D4, has the most shielding due to the surrounding detectors and yielded the lowest counting rate. However, the complex shielding geometry makes interpretation of the single-detector counting rates difficult, because they do not correspond directly to incident flux. We have therefore performed laboratory calibrations using an identical telescope to those flown on the two Voyager spacecraft. Results from D1 will be of primary concern due to that detectors higher sensitivity, although the calibration of D2 will also be of interest. D1 has the additional advantage of providing pulse height analysis of a small fraction of its recorded events, and these will be essential in deriving spectral information.

The aim of the calibrations is to allow a calculation of the electron differential intensity, $j(E, \alpha)$, which is a function of kinetic energy, $E$, and pitch angle, $\alpha$. Here we assume that the

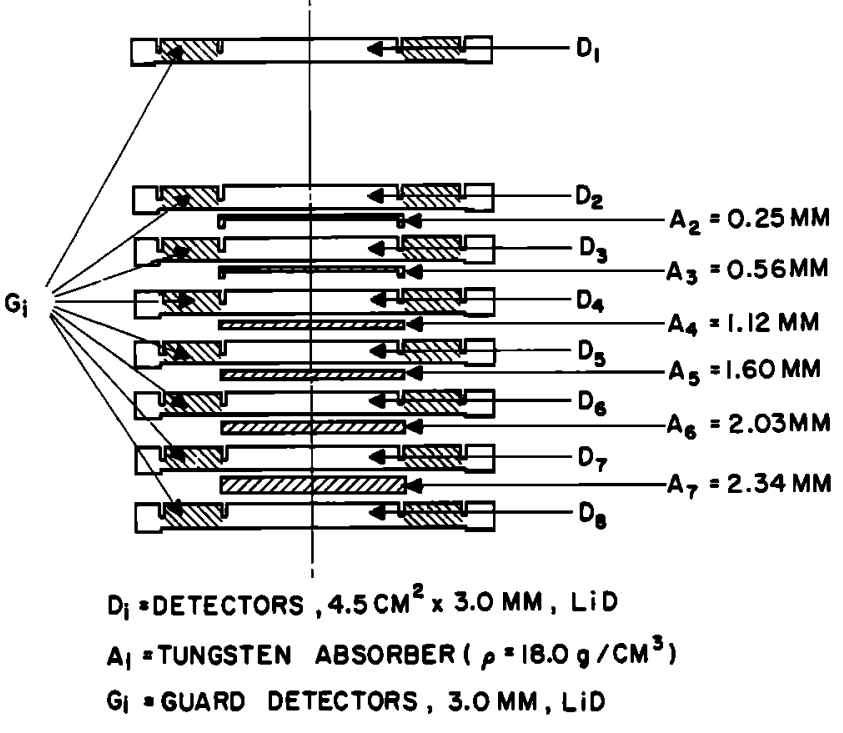

\section{THE ELECTRON TELESCOPE} (TET)

Fig. 1. Schematic of the detector layout in the CRS electron telescope (TET).

magnetospheric electrons are gyrotropic with respect to the local magnetic field. Referring to a coordinate system with its polar axis along the symmetry axis of the telescope, $\alpha$ is a function of the spherical angles $\theta$ and $\phi$. The response function, $R$, of a given detector is defined by

$$
N(E)=\tau \int_{0}^{\infty} \int_{0}^{2 \pi} \int_{0}^{\pi} R\left(E, E^{\prime}, \theta\right) j\left(E^{\prime}, \alpha(\theta, \phi)\right) \sin \theta d \theta d \phi d E^{\prime}
$$

where $N(E) d E$ is the number of electrons depositing energies between $E$ and $E+d E$ in the detector during the livetime $\tau$. Azimuthal symmetry in $R$ is assumed, corresponding to that of the telescope. The measurements of $R$ by laboratory calibration are described in the appendix.

There are two types of data from D1. The integral counting rates are formed by integrating $N(E)$ over all energies, $E$, above the electronic threshold of $E=0.5 \mathrm{MeV}$. The pulse height analyzed (PHA) data are formed by integrating $N(E)$ over the energy range of each analyzer channel (which have widths of about $20 \mathrm{keV}$ ). The integrations lead to integral response functions, $R_{i}\left(E^{\prime}, \theta\right)$, where $i$ labels the channel number. By including the entire energy range applicable to the integral counting rate as one extra channel labeled by a particular value of $i$, the entire data set is represented as a set of $N_{i}$. From (1), allowing for a channel dependent livetime $\tau_{i}$,

$$
N_{i}=\tau_{i} \int_{0}^{-2 \pi \pi} \int_{\theta}^{\pi} R_{i}\left(E^{\prime}, \theta\right) j\left(E^{\prime}, \alpha(\theta, \phi)\right) \sin \theta d \theta d \phi d E^{\prime}
$$

The set of $N_{i}$ can also include data from other detectors by integrating the appropriate response functions. The data analysis consists of inverting (2) to find $j(E, \alpha)$.

The extent to which $j(E, \alpha)$ can be resolved by $R_{i}\left(E^{\prime}, \theta\right)$ from the data, $N_{i}$, is determined by the extent to which $R$ approximates a delta function corresponding to the stopping energy of the electron. At higher energies more of the electrons penetrate the detector, resulting in a peak in $R$ corresponding to minimum ionization that is essentially independent of electron energy. As a result, the spectral sensitivity of (2) is limited to $E^{\prime} \leq 2.5 \mathrm{MeV}$. 
The response function introduces some nonuniqueness into the determination of $j$. We have therefore chosen to parameterize $j$ with the function

$$
j(E, \alpha)=A_{0} E^{-\gamma} \sin ^{2 n} \alpha_{0}
$$

where $A_{0}, \gamma$ and $n$ are constants. The subscript 0 refers to equatorial values, which are related to local values (no subscript) on the same magnetic field line by

$$
\begin{gathered}
\sin ^{2} \alpha=\frac{B}{B_{0}} \sin ^{2} \alpha_{0} \\
A=\left[\frac{B_{0}}{B}\right]^{n} A_{0}
\end{gathered}
$$

where $B$ is the magnitude of the magnetic field. Equation (4a) follows from conservation of energy and the first adiabatic invariant (magnetic moment) and $(4 b)$ follows from Liouville's theorem on an equipotential field line. The pitch angle dependence in (3) is convenient because it is maintained at different locations on a given magnetic field line and because it is a simple representation of the typical trapped ("pancake" shaped) distribution function.

The model form (3) for $j$ allows a calculation of a simulated data set, $\hat{N}_{i}$, via (2). The three parameters $A_{0}, \gamma$ and $n$ are then adjusted to obtain the best fit to the observed data, $N_{i}$. Since the data are collected in a counting experiment, they obey Poisson statistics and the best fit is defined by the minimization of the Poisson likelihood $\chi^{2}$ function [Baker and Cousins, 1984]

$$
\chi_{p}^{2}=2 \sum_{i}\left[\tilde{N}_{i}-N_{i}+N_{i} \ln \left[\frac{N_{i}}{\tilde{N}_{i}}\right]\right]
$$

This has the same interpretation as the least squares $\chi^{2}$ function has for Gaussian statistics.

In practice it was not necessary, for the accuracy required, to perform the solid angle integration in (2) at every iteration of the fit procedure. We have therefore chosen three values, $n=0,1$ and 2 , for the anisotropy parameter, and $\theta_{B}=0,45^{\circ}$ and $90^{\circ}$, for the angle from the TET telescope axis to the local magnetic field, and tabulated the solid angle integrations at these values. The fit procedure then uses a linear interpolation between the tabulated values. The results of the integrations are close enough to linear with respect to $n$ and $\theta_{B}$ for this procedure to provide accurate results. In addition, we have made corrections to the simulated data for the effects of detector deadtime and pulse pileup which are described in the appendix.

In applying the procedure described above to a data set taken by CRS near a single point in space, the anisotropy parameter, $n$, is generally not constrained. That is, the uncertainty in $n$ is unacceptably large due to the nearly omnidirectional nature of the detector response functions. We have therefore chosen to simultaneously fit two data sets taken at the same $L$ value during the inbound and outbound portions of the spacecraft trajectory, assuming that the values of the three fit parameters are constant on a given $L$ shell. The value of $n$ is then constrained by the variation of electron flux with magnetic latitude. A sample fit is shown in Figure 2. The PHA data, shown by the two histograms which have been binned with four PHA channels per bin, were taken over time intervals of approximately $5 \mathrm{~min}$ each, centered at 13:12 (top) and 21:49 SCET (spacecraft event time) on day 24.

In addition to the values of the fit parameters, we also wish to know the range of energies over which they are applicable. The energy range is not simply given by the PHA channels which

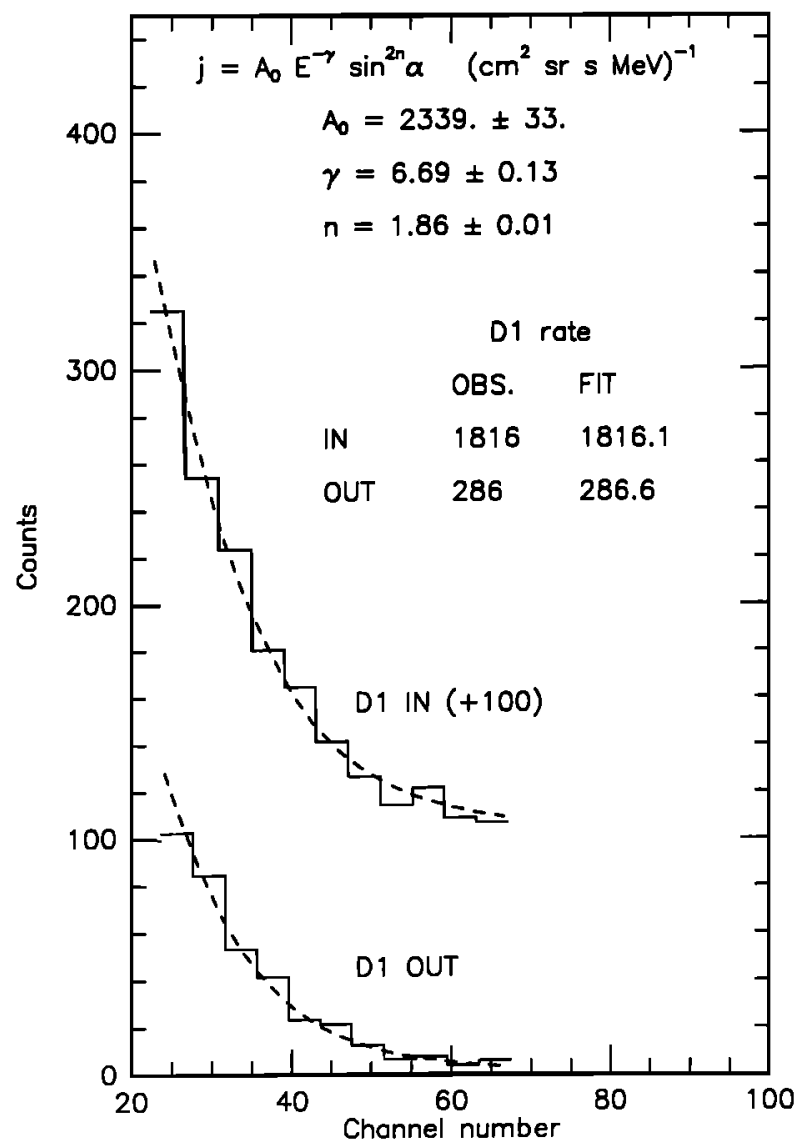

Fig. 2. A sample fit to data from Uranus. The two histograms show the PHA data taken at 13:12 SCET inbound (top, +100 counts) and 21:49 SCET outbound between $L$ values of 11.8 and 12.2 (the range is smaller at lower $L$ ). The dashed curves are the simulated PHA data from the fit. The two D1 counting rates are shown above with the simulated rates from the fit. At the top are the model equation and the three fit parameters with their statistical uncertainties.

were included in the fit because of the energy loss in the passive shielding around the detector and the additional information contained in the integral counting rates. The energy range, and how well $j$ is approximated by a power law, are found from a direct inversion. By discretizing $j,(2)$ converts into a simple linear matrix equation which we solve using the generalized inverse approach of Tarantola and Valette [1982] (the approach strictly applies only to least squares problem but is adequate for the present purpose). One spectrum is fit using the pitch angle dependence from the inbound/outbound power law fit. To constrain the inversion, the best fit power law is used as prior information with an arbitrarily large uncertainty (typically $100 \%$ ) and some correlation between adjacent points is also included. The procedure is illustrated in Figure 3. Points on the spectral fit where the error bars are small compared with the prior value are said to be well resolved. These occur between energies of $\sim 0.7$ and $2.5 \mathrm{MeV}$, which is typical of all of the cases studied and therefore provides a good estimate of the energy range over which the analysis applies.

Figure 3 also contains three points from the measurements made by the low-energy charged particle (LECP) experiment [Mauk et al., 1987]. These are from the LECP channels which have energy ranges from 112 to $183 \mathrm{keV}, 252$ to $480 \mathrm{keV}$ and 480 to $853 \mathrm{keV}$, and are plotted at the geometrical mean of each 

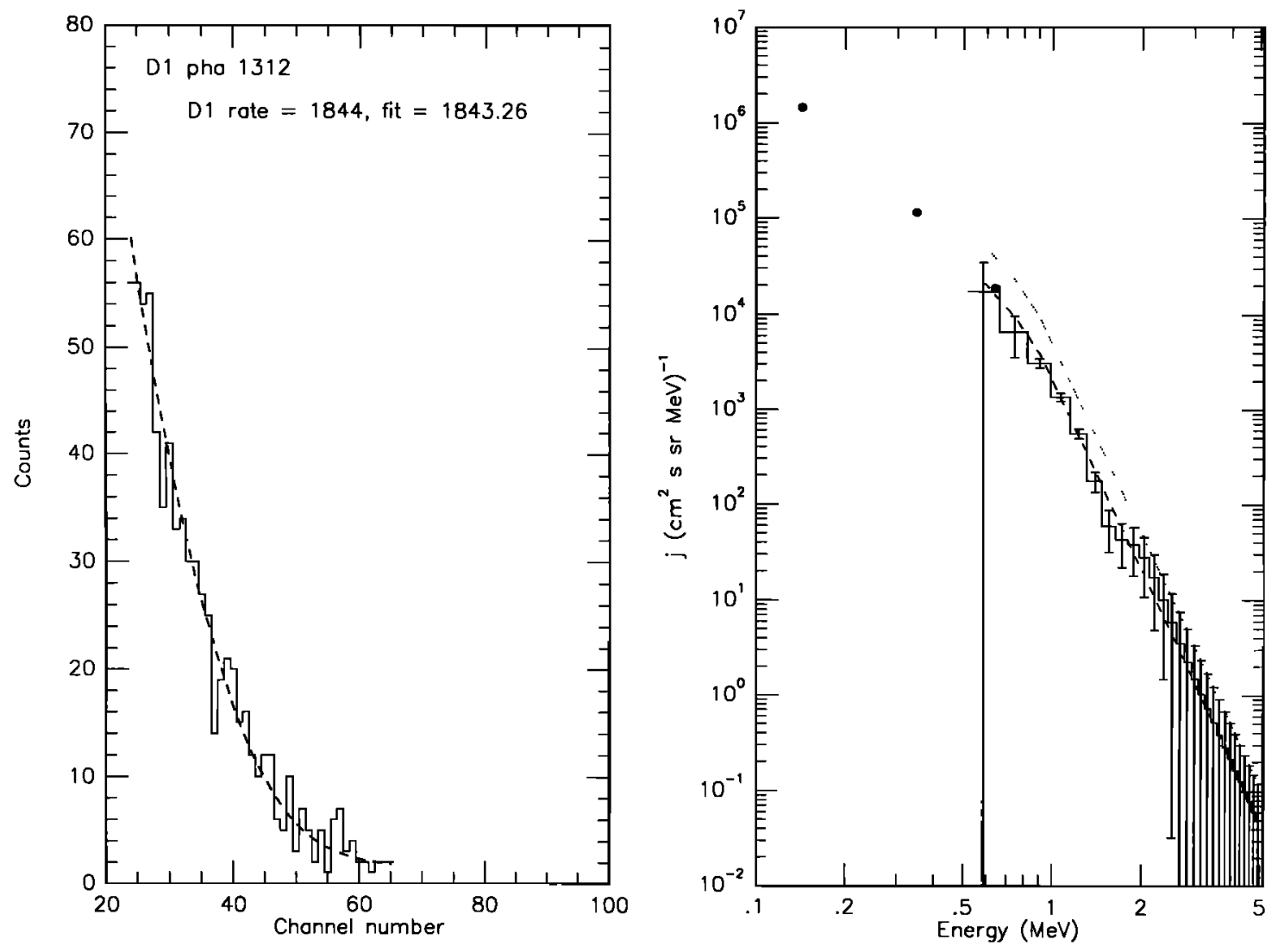

Fig. 3. A sample fit using the discrete inversion technique described in the text. (Left) The inbound data from 13:12 SCET is shown, with the same format as Figure 2, except that the PHA channels are now unbinned. (Right) Differential intensity versus energy. The histogram with error bars shows the result of the fit. The dashed curve is the best fit power law from Figure 2 which is used here as prior information. The dotted curve is the $1 \sigma$ upper limit on the prior information. The three points at low energies are from the LECP experiment.

range. The LECP and CRS spectra are continuous, but there is a change in the power law index, $\gamma$, evident from the change in slope on the log-log plot. We have therefore included a change in $\gamma$ in the power law fits, using the values given by Mauk et al. [1987] for the three LECP channels below $850 \mathrm{keV}$ and leaving it as a free parameter above $850 \mathrm{keV}$. The value used in the Figure 2 fit below $850 \mathrm{keV}$ was $\gamma=3.4$ and is evident by close inspection of the dashed curve in Figure 3. This procedure gives us some confidence in extropolating the results to somewhat lower energies than the $0.7 \mathrm{MeV}$ discussed above.

The observed D1 and D2 integral rates are plotted versus $L$ in Figure 4. They are averaged over each time interval for which a fit to the spectrum has been made. The inbound (heavy curve) and outbound intervals have been chosen so that the centers of each pair are as close as possible to the same $L$, to the extent allowed by the time resolution of the data. The lengths of the time intervals were chosen to give enough counts for low statistical uncertainties in the PHA data, while still being sufficiently short to resolve the time variations in the data. The points in Figure 4 for which fits have actually been made are between $L=6.6$ and 14.7. Inside this region the high fluxes cause the detector response to become too nonlinear for a reliable analysis, due to unacceptably large deadtime and pileup corrections (see appendix). Inside $L=10$ the D2 integral rate was high enough that it was included in the analysis with the D1 integral rate and PHA data.

\section{RESULTS}

\section{Fit Parameters}

The three fit parameters, $A_{0}, \gamma$ and $n$, are plotted versus $L$ in Figure 5 . Statistical errors are not shown because they are generally small due to the high counting rates. Systematic errors are probably larger and may be due to calibration error in the detector response functions or inaccurate parameterization of the model intensity function. We estimate that the calibration error is $\leq 10 \%$ and that the parameterization is probably the dominant potential source of error. To evaluate this possibility we have repeated the analysis using a substantially different model for the pitch angle dependence, in which $j$ is constant above some pitch angle (the "loss cone" angle) and zero below. The loss cone angle replaces $n$ as a fit parameter. The results showed that this model was highly artificial because the flux always dropped to zero just above the highest spacecraft magnetic latitude. Even so, the phase space densities (see below) derived from this model were not significantly different from those derived with the more realistic model, and we therefore consider that systematic errors introduced by the model do not alter any of the conclusions discussed in the next section.

A convenient way to view the results is provided by the polar $R-\Lambda$ magnetic coordinate system [e.g., Roederer, 1970], defined by

$$
R=L \cos ^{2} \Lambda
$$




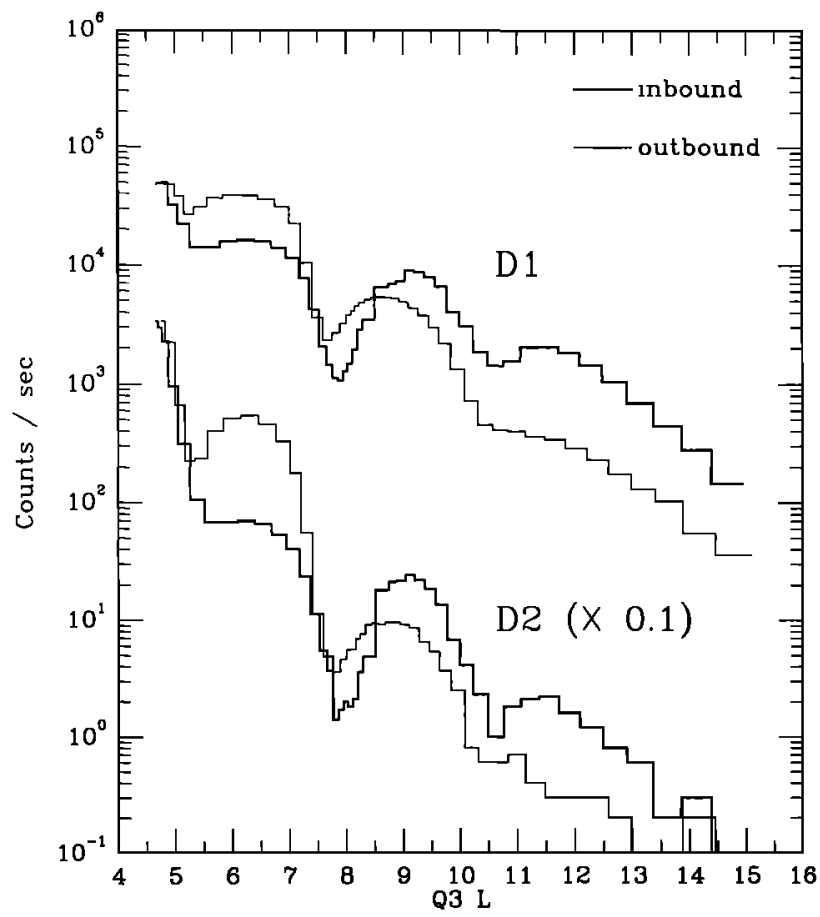

Fig. 4. D1 and D2 counting rates, averaged over the time intervals used in the fits, versus $L$.

$$
B=\frac{k_{0}}{R^{3} R_{U}^{3}}\left(4-\frac{3 R}{L}\right)^{\frac{1}{2}}
$$

where $k_{0}=0.23 \mathrm{GR} R_{U}^{3}$ is the planetary magnetic dipole moment and $R_{U}$ is the radius of Uranus $(25,600 \mathrm{~km}) . \Lambda$ is the invariant latitude. These coordinates provide a way of projecting integral flux data from an arbitrary magnetic field, such as the Q3 model used here, to a dipole geometry in a single meridian. To carry this out we use the model differential flux from each fit to calculate the omnidirectional integral flux as a function of $B$ along the Q3 field line. Then, for given $R$ and $\Lambda$ we convert to $B$ and $L$ with (6) and use linear interpolation to find the flux at that point. A color-coded $R-\Lambda$ flux map for the omnidirectional integral flux above $1 \mathrm{MeV}$ is shown in Plate 1.

The spacecraft trajectory in $R-\Lambda$ coordinates is shown in Figure 6, with points along the trajectory indicating the locations that correspond to the data points used in the analysis. There is one inbound and one outbound point (solid circles) for each $L$. Also shown are dipole magnetic field lines with $L$ equal to the approximate orbital minimum values for Miranda (5.1), Ariel (7.5) and Umbriel (10.4) [Acuna et al., 1988]. The dashed curves show the locations of the mirror points for electrons with indicated values of the second adiabatic invariant, $K$ (see below).

\section{Phase Space Densities}

The phase space density is useful for considering the sources, sinks and diffusive transport of electrons. It is related to the differential intensity by

$$
f(\mathbf{r}, \mathbf{p})=\frac{j(E, \alpha)}{p^{2}}
$$

where $r$ is position, $p$ is momentum and $p=|p|$. Equation (7) is also appropriate, within a constant, to the density in a space of the

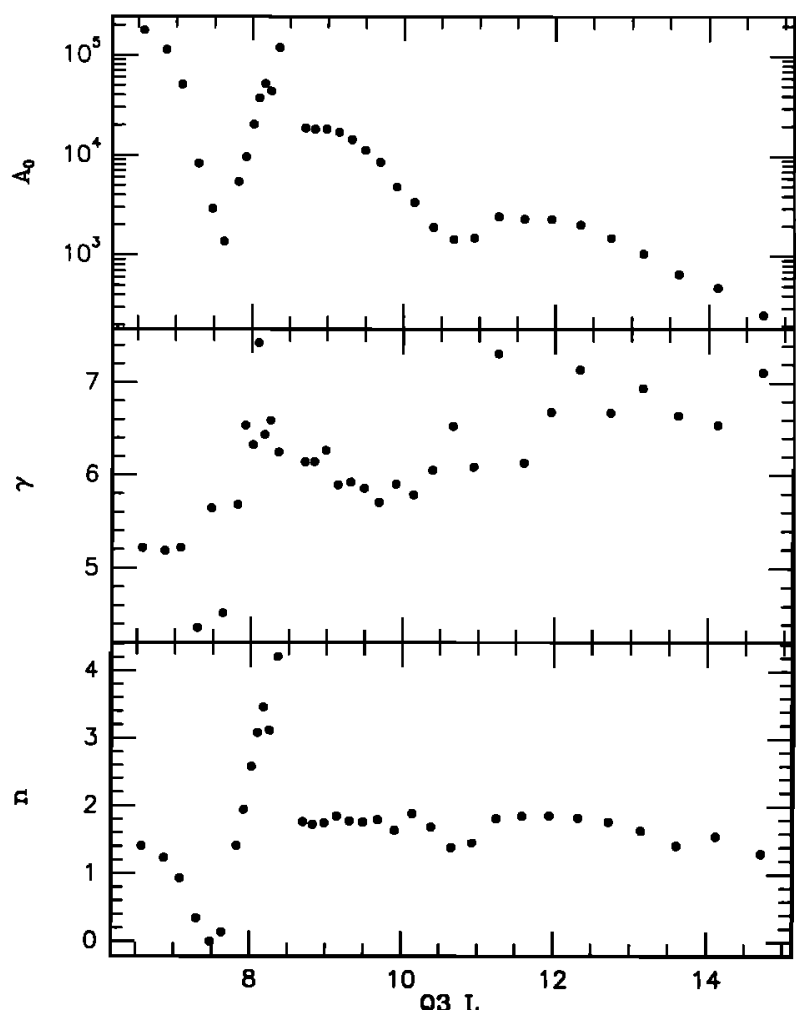

Fig. 5. The fit parameters, $A_{0} \gamma$ and $n$ versus $L$. The units of $A_{0}$ are $\left(\mathrm{cm}^{2} \mathrm{sr} \mathrm{s} \mathrm{MeV}^{-1}\right)$.

three adiabatic invariants, $M, J$ and $\Phi$, because there exists a canonical transformation between the phase space coordinates and the canonical variables which are proportional to the adiabatic invariants [Schulz and Lanzerotti, 1974]. Then $f$ satisfies the diffusion equation [Haerendel, 1968]

$$
\frac{\partial f}{\partial t}=\frac{1}{G} \nabla \cdot(G \mathrm{D} \cdot \nabla f)+S
$$

where $D$ is the diffusion tensor, $S$ is the net source of electrons and $G$ is the Jecobian of the transformation from $M, J$ and $\Phi$ to the coordinates in use.

The first adiabatic invariant (magnetic moment) is

$$
M=\frac{p^{2}}{2 m B_{m}}
$$

where $m$ is the electron rest mass and $B_{m}$ is the magnetic field at the mirror points. In the case of equipotential field lines the second adiabatic invariant $J=2(2 m)^{1 / 2} K$, where

$$
K=\int_{l_{1}}^{s_{2}}\left(B_{m}-B\right)^{1 / 2} d s
$$

$s_{1}$ and $s_{2}$ are the mirror point positions and the integral is along the guiding center magnetic field line. The third adiabatic invariant, $\Phi$, which is the magnetic flux inside a drift shell, is related to the invariant shell parameter, $L^{*}$, by [e.g., Roederer, 1970]

$$
L^{*}=\frac{2 \pi k_{0}}{R_{U} \Phi}
$$

Since drift shell splitting is negligible, we expect that $L^{*}$ can be 


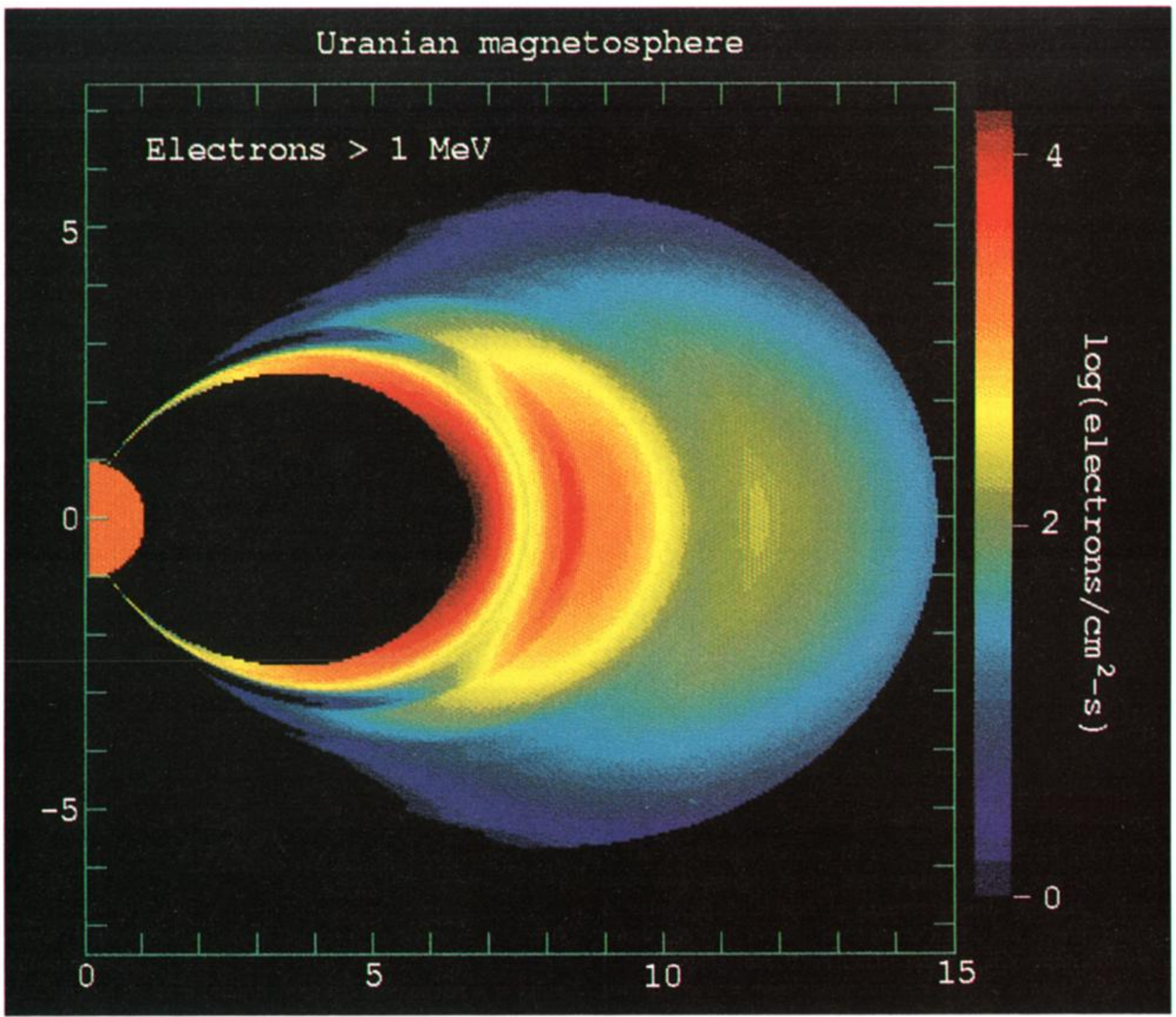

Plate 1. Omnidirectional flux map in polar $R-\Lambda$ coordinates for electrons above $1 \mathrm{MeV}$. The units are indicated by the color bar on the side.

replaced by $L$ and then (7) and (8) apply in the convenient coordinates $M, K$ and $L$.

In radial diffusion $K$ and $M$ are conserved. The diffusion velocity is then proportional to the radial gradient of $f$ at constant $K$ and $M$. To compute $f$ at given values of $K$ and $M$ we proceed as follows:

1. At each $L$ where model fits to the data have been made, compute $B_{m}$ by numerically solving

$$
\frac{L^{3} R_{U}^{3} B_{m}}{k_{0}}=F\left[\frac{K^{3}}{B_{m}^{1 / 2} k_{0}}\right]
$$

where $F$ is the function which defines $L$ in terms of $K$ and $B_{m}$ [Mcllwain, 1966].

2. Compute $\alpha_{0}$ from (4a), $p$ from (9) and convert $p$ to kinetic energy, $E$.

3. From (3) calculate $j$ and finally $f$ from (7).

Plots of $f$ versus $L$ for different values of $K$ and $M$ are shown in Figures 7 to 10. The points marked with a solid circle are within the CRS energy range of 0.7 to $2.5 \mathrm{MeV}$ discussed above. Points with energies below this range are marked with an open circle and those above with a cross. Note that the kinetic energy for electrons at constant $K$ and $M$ is a decreasing function of $L$. Also shown are smooth curves corresponding to each pair of $K$ and $M$ values. These were obtained by a least squares fit of a solution to the radial diffusion equation and are described in detail in the next section.

\section{IMPUICATIONS FOR DIFFUSION}

The dominant features of the results described above are the deep local minima in the phase space density near the minimum $L$ values of Ariel and, to a lesser extent, Umbriel. These are consistent with the similar features in the raw counting rate data [Cooper and Stone, 1991]. The satellite absorption efficiency is strongly peaked near the minimal $L$ of the orbit [Paonessa and Cheng, 1987], so the features are macrosignatures, representing the average effect of satellite sweeping over many orbits. If satellite losses are replenished primarily by diffusion, then the structure of the phase space density profiles and the absorption efficiency provide an opportunity to study the diffusion process. 


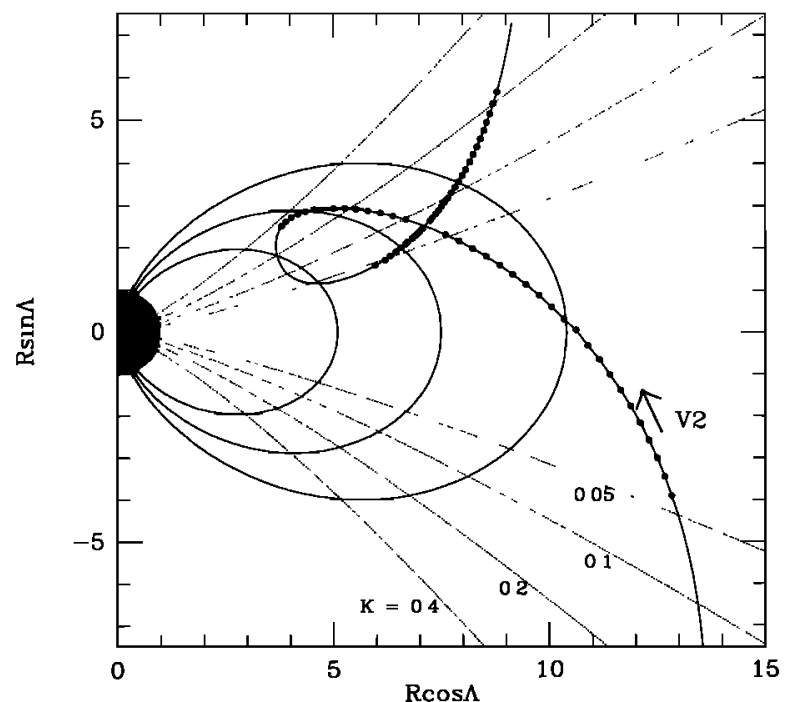

Fig. 6. Voyager 2 trajectory in $R-\Lambda$ coordinates. The points along the trajectory show the locations of the data to which fits have been made. The dashed curves are the mirror point locations for electrons with the indicated $K$ values. The dipole field lines correspond to the minimum $L$ values of Miranda, Ariel and Umbriel.

A model for the effective lifetime of electrons against satellite sweeping, $\tau_{s s}$, is given in the appendix. The model differs from that of Paonesse and Cheng [1987] and Cooper [1990] because we have taken into account that the range $\Delta L$ corresponding to the satellite radius $\left(r_{\mathrm{sut}}\right)$ depends on the magnetic latitude. The previous authors assumed that $\Delta L=r_{\text {sat }}$ which results in a significant overestimate of $\tau_{\mathrm{ss}}$ when the satellite is at high magnetic latitudes (this may not be true for energetic ions where corrections for the large gyroradii are required). The model applies only to dipole magnetic fields, so in this section we use the OTD field model with dipole $L$ corresponding to the Q3 $L$ values of the previous sections. As noted previously, both the OTD and Q3 models lead to similar offsets of the absorption features from the satellite minimum $L$ values.

Sample values of $\tau_{s s}$ are shown in Figure 11 versus OTD $L$ for $M=2000 \mathrm{MeV} \mathrm{G}^{-1}$ and for three values of $K$. The lifetime is shortest near the satellite minimum $L$ where the $L$ shell contact time is longest and the satellite is near the magnetic equator. At larger $L$ the satellite moves to higher magnetic latitudes where electrons with large equatorial pitch angles mirror below the satellite and are not absorbed. Therefore the higher $K$ values are absorbed over a larger range of $L$ outside each minimum $L$.

The variation of $\tau_{s s}$ with $K$ means that electrons which diffuse radially past a satellite sweeping region will suffer a dramatic change in their pitch angle distribution as smaller pitch angles (larger $K$ ) are preferentially absorbed. Inside the satellite minimum $L$, where absorption cuts off at all pitch angles, the pitch angle distribution should remain in the highly anisotropic form which is strongly peaked at $90^{\circ}$. The anisotropy parameter, $\boldsymbol{n}$, in Figure $\mathbf{5}$ shows that there is a rapid change in anisotropy near the minimum $L$ of Ariel and a small change near the minimum $L$ of Umbriel, but that away from these regions $n$ has a nearly uniform value of $n \approx 1.7$, rather than steadily increasing inward. These results lead us to suggest that together with satellite sweeping and radial diffusion there is significant pitch angle diffusion which is sufficient to maintain the nearly uniform pitch angle anisotropy. The distribution functions expected with and without pitch angle diffusion are illustrated by a quantitative model below.

The existence of minima in the phase space density, rather than just changes in slope, is also unexpected in the presence of satellite sweeping and radial diffusion alone, requiring a local source of energetic electrons. In the present context, the requirement for a local source includes the possibility of an effective source at a given $K$ and $M$ due to nonconservation of $K$

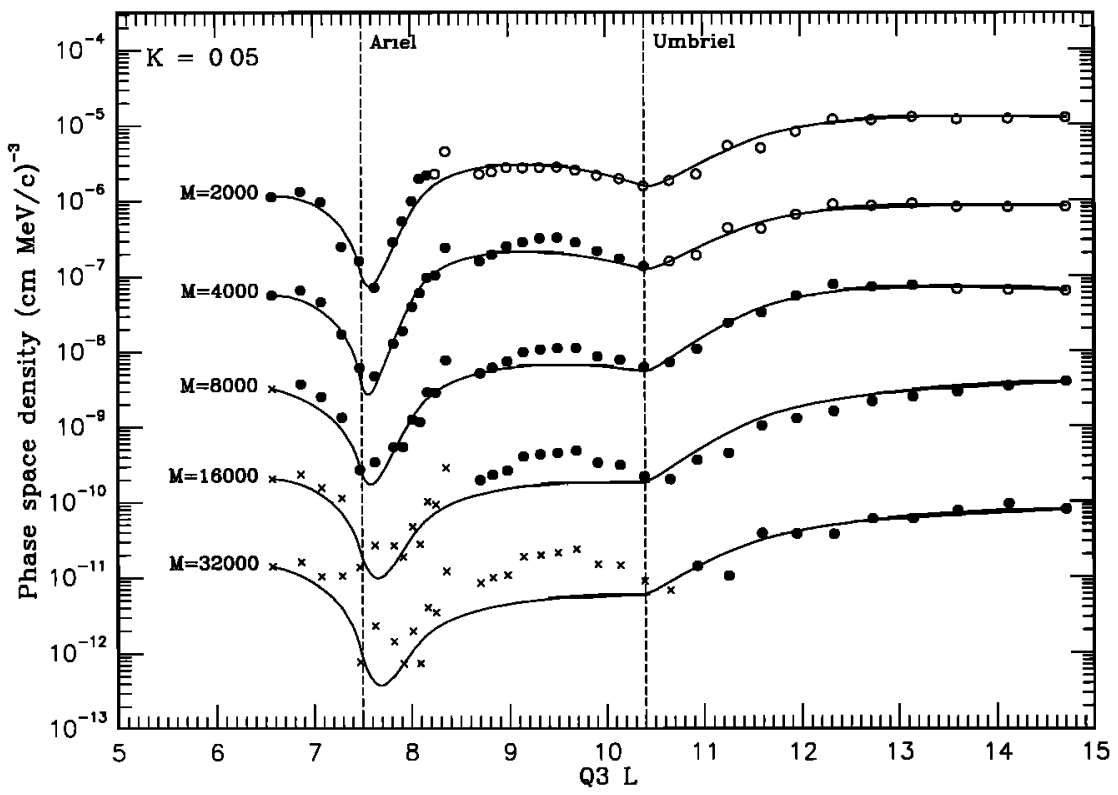

Fig. 7. Phase space densities for $K=0.05 \mathrm{G}^{1 / 2} R_{U}$ as a function of $L$ and $M$. The units of $M$ are $\mathrm{MeV} \mathrm{G}^{-1}$. The solid circles correspond to kinetic energies in the range from 0.7 to $2.5 \mathrm{MeV}$. The open circles (crosses) are for energies below (above) this range. Each pair of $K$ and $M$ values has a smooth curve through the boundary points which represents a fit of solutions of a model diffusion equation to the interior points. 


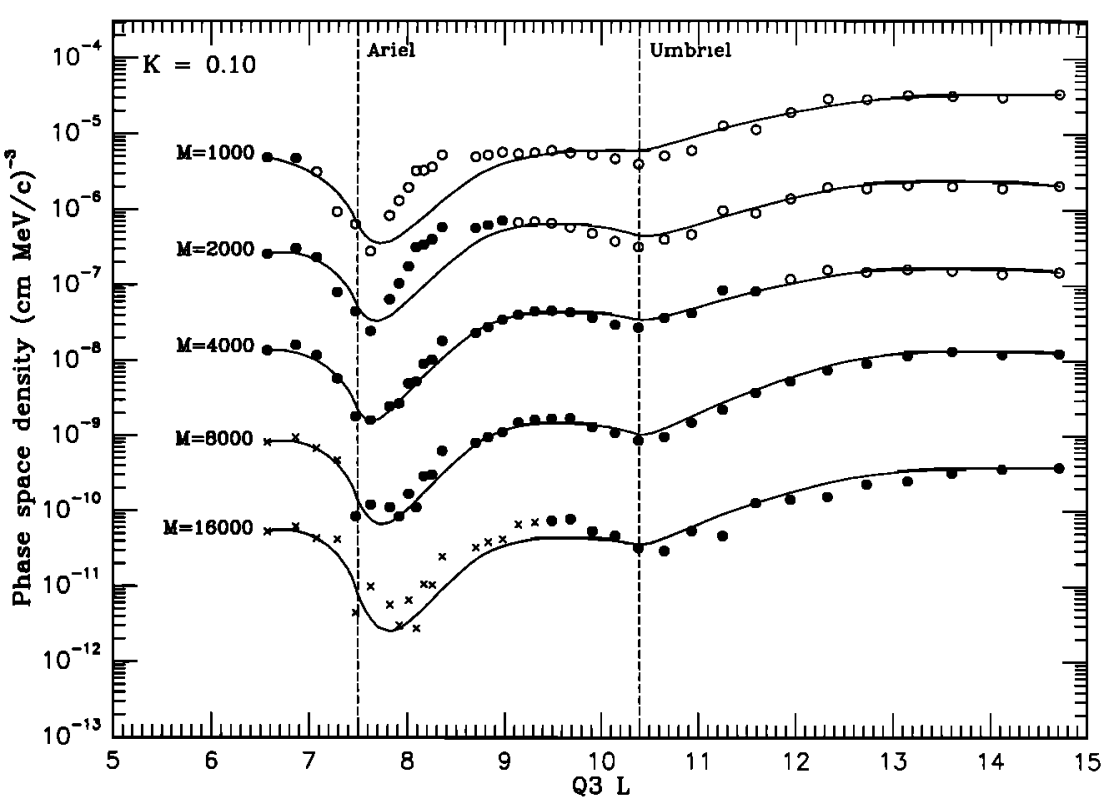

Fig. 8. Same as Figure 7 but for $K=0.1 \mathrm{G}^{1 / 2} R_{U}$.

and/or $M$. If there exists a local source sufficient to increase the phase space density inside the satellite orbits and produce the observed minima, then it is possible that the nearly uniform anisotropy could result from radial diffusion alone. However, the diffusion coefficient would have to increase with $K$ (decrease with equatorial pitch angle) at a specific rate determined by the $K$ dependance of $\tau_{s s}$, which is unlikely. The apparent isotropization at the minimum $L$ value of Ariel reflects the $K$ dependence of the locations in $L$ of the phase space density minima, which occur at slightly higher $L$ for higher $K$ values.

Pitch angle diffusion at constant $L$ violates the first two adiabatic invariants, $M$ and $K$. Therefore bimodal diffusion in $L$ and pitch angle violates all three adiabatic invariants simultaneously. To avoid solving the full diffusion equation in three dimensions, Walt [1970] introduced a new variable $\zeta=M / y^{2}$, where $y=\sin \alpha_{0}$, which is conserved in pitch angle diffusion at constant energy and approximately conserved in radial diffusion. Under this approximation, the diffusion tensor in the coordinates $L, x=\cos \alpha_{0}$, and $\zeta$, has the form

$$
\mathbf{D}=\left[\begin{array}{ccc}
D_{L L} & 0 & 0 \\
0 & D_{x x} & D_{\zeta \times} \\
0 & D_{x \zeta} & D_{\zeta \zeta}
\end{array}\right]
$$

Pitch angle scattering is often nearly elastic [Schulz and Lanzerotti, 1974], so that the $\zeta$ diffusion coefficients, $D_{\zeta \zeta}$ and $D_{x \zeta}=D_{\zeta_{x}}$ are small. If they are ignored then, with the Jacobian (within numerical factors) $G=\left(\zeta / L^{5}\right)^{1 / 2} x T(y)$, the time

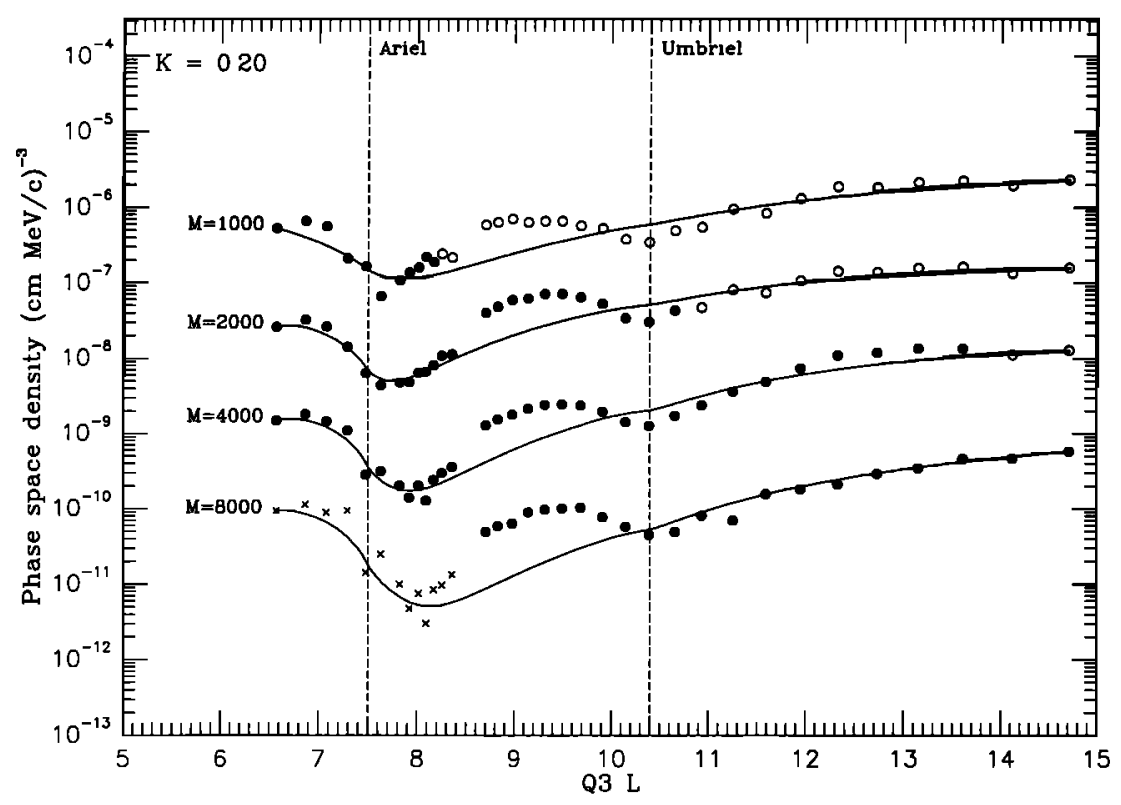

Fig. 9. Same as Figure 7 but for $K=0.2 G^{1 / 2} R_{U}$. 


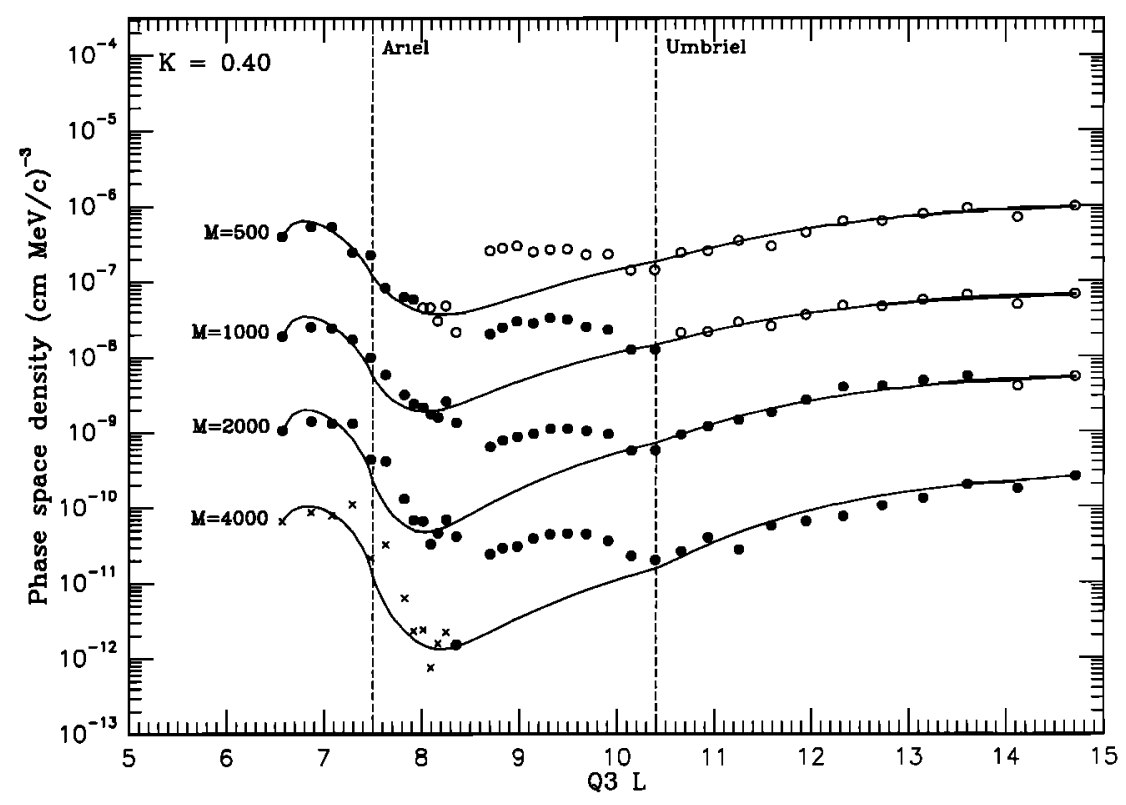

Fig. 10. Same as Figure 7 but for $K=0.4 G^{1 / 2} R_{U}$.

independent diffusion equation from (8) becomes

$$
L^{5 / 2} \frac{\partial}{\partial L}\left[L^{-5 / 2} D_{L} \frac{\partial f}{\partial L}\right]+\frac{1}{x T(y)} \frac{\partial}{\partial x}\left[x T(y) D_{x x} \frac{\partial f}{\partial x}\right]=\frac{f}{\tau}
$$

where $T(y)=1.38-0.32\left(y+y^{1 / 2}\right.$ ) [Schulz and Lanzerotti, 1974]. The lifetime, $\tau$, includes $\tau_{s s}$ and any other sources or losses that are proportional to $f$. One way to solve (14) [Walt, 1970] is to expand $f$ in a series of pitch angle eigenmodes $g_{n}(x)$, $f=\sum_{n} a_{n}(L, \zeta) g_{n}(x)$, where

$$
\frac{1}{x T(y)} \frac{\partial}{\partial x}\left[x T(y) D_{x x} \frac{\partial g_{n}}{\partial x}\right]=-\lambda_{n}(L, \zeta) g_{n}(x)
$$

If the $y$ dependence of $T$ is ignored and $D_{x x}$ is independent of $x$ then [Schulz and Lanzerotti, 1974] $g_{n}(x)=2^{1 / 2} J_{0}\left(k_{n} x\right) / J_{1}\left(k_{n}\right)$ with eigenvalues $\lambda_{n}=D_{x x} k_{n}^{2}$. $J_{0}$ and $J_{1}$ are Bessel functions of order zero and one respectively and $J_{0}\left(k_{n}\right)=0$ (the loss cone is assumed to be at $x=1$, so $g_{n}(1)=0$, which is a good approximation for the $L$ range of interest). By expanding $f$ in (14) and then substituting the eigenvalue condition (15), the coefficients $a_{n}$ are found by the orthogonality of the $g_{n}$ to satisfy a set of linear ordinary differential equations

$$
L^{5 / 2} \frac{\partial}{\partial L}\left[L^{-5 / 2} D_{L} \frac{\partial a_{n}}{\partial L}\right]-\lambda_{n} a_{n}=\sum_{m} \tau_{m m}^{-1} a_{m}
$$

where the matrix

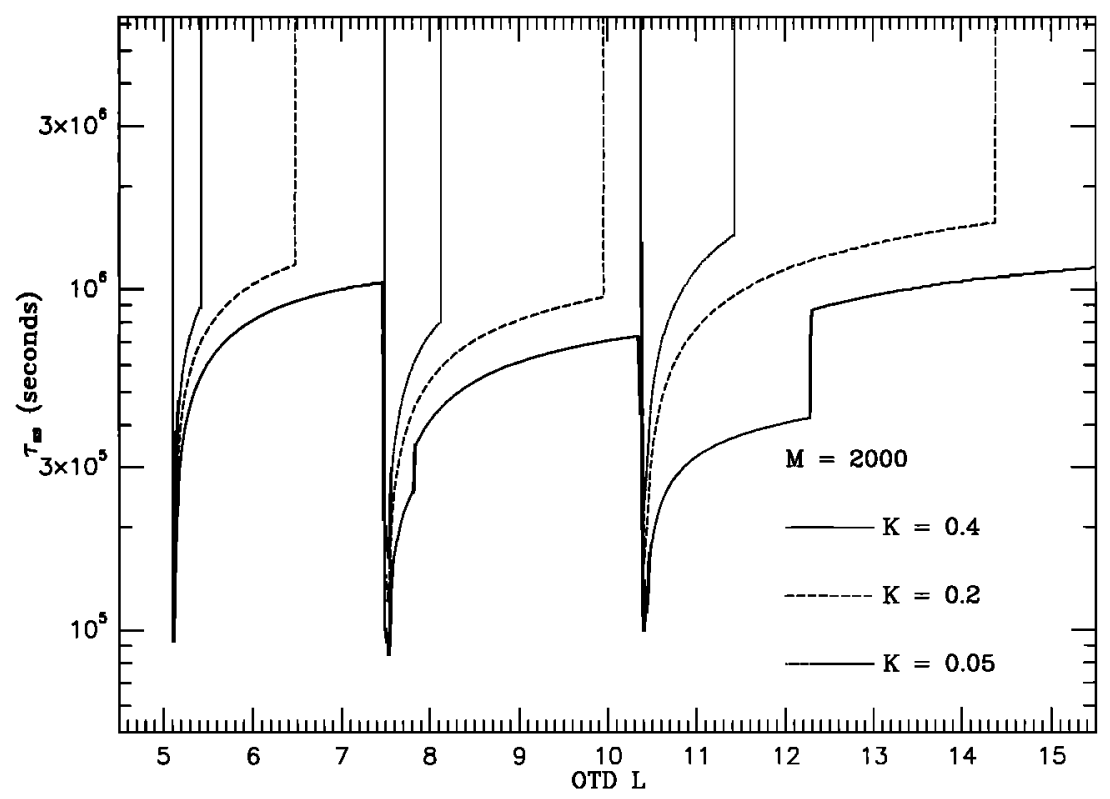

Fig. 11. Satellite absorption lifetimes for $M=2000 \mathrm{MeV} \mathrm{G}^{-1}$ and $K=0.05,0.2$ and $0.4 \mathrm{G}^{1 / 2} R_{U}$. The discontinuities in the $K=0.4$ curve are due to overlap of the absorption regions. 


$$
\tau_{m}^{-1}=\int \frac{1}{\tau(x)} x g_{n}(x) g_{m}(x) d x
$$

and $D_{L}$ is independent of $x$.

The $a_{n}$ are the amplitudes of the pitch angle eigenmodes which, if $\tau$ is independent of $x$, diffuse independently. The $\lambda_{n}$ are inverse lifetimes of the eigenmodes which decay faster with increasing $n$. If pitch angle diffusion were the only process occurring then, because all of the $\lambda_{n}$ are positive, the eigenmode amplitudes would all decrease with time, the decay representing particle diffusion into the loss cone and subsequent precipitation into the atmosphere of the planet. The precipitation loss rate increases with $D_{x x}$ and with the amplitudes of the high-order eigenmodes relative to lowest order. Since the lowest order eigenmode has the longest lifetime, it should be the most commonly observed unless the higher order modes are being locally excited. The Bessel function representation of the lowest pitch angle eigenmode is well approximated by $y^{2 n}$ with $n=1.4$. Therefore it is a good approximation to the pitch angle distribution derived from the data away from the satellite signatures, indicating that $D_{x x}$ is approximately independent of $x$ as assumed.

The $x$ dependence of $\tau_{s s}$ in the satellite absorption regions couples diffusion in the different eigenmodes and causes the higher order modes to be excited. Thus high order eigenmodes will have their largest amplitudes in the absorption regions and pitch angle diffusion can then act as a source of particles at intermediate pitch angles. However, the higher order modes also have shorter lifetimes so that satellite absorption increases the net loss of particles by precipitation.

Given forms for the diffusion coefficients and lifetime $\tau$, the radial equation (16) is solved numerically for the set of $a_{n}$ by a finite difference, sparse matrix inversion technique [Press et al., 1986]. Boundary conditions are specified by requiring that all of the $a_{n}=0$ at $L=1$ and that only the lowest order coefficient, $a_{1}$, be nonzero at an outer boundary. The series is truncated at $n=6$ which was found to provide stable solutions that are accurate except for $1-x<1$. For simplicity, only absorption by Ariel with its minimum $L$ at 7.5 is included at this stage and the outer boundary is at $L=14$.

Three sample solutions are given in Figure 12, which shows contours of $f$ versus $L$ at equally spaced values of $x$ from 0 to 0.8 , and for $\zeta=8000 \mathrm{MeV} \mathrm{G}^{-1}$. A solution with no pitch angle diffusion, $D_{x x}=0$, is shown in Figure $12 a$. Here the effect of the satellite sweeping in exciting high-order pitch angle eigenmodes is illustrated. The pitch angle anisotropy increases as electrons

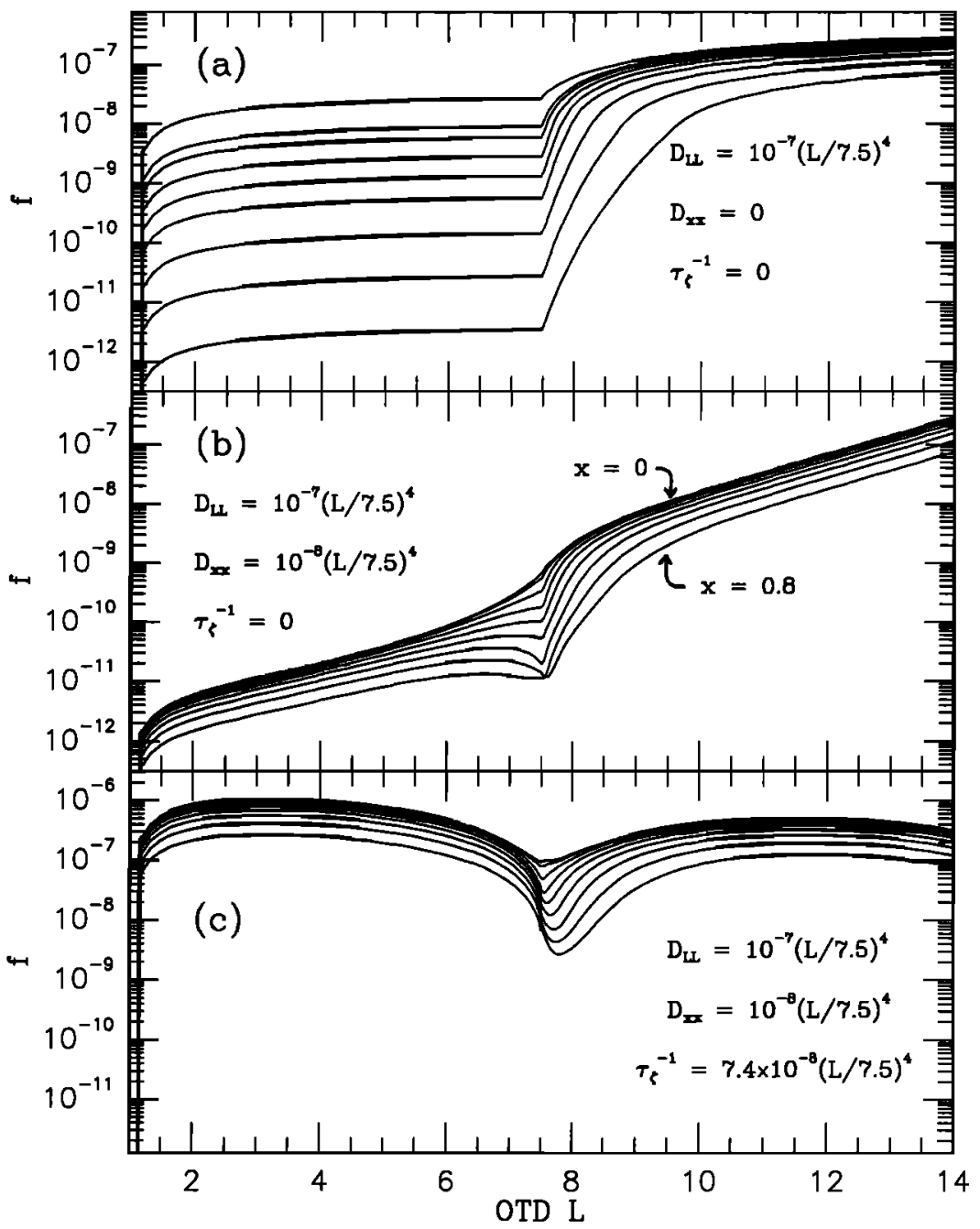

Fig. 12. Solutions of the bimodal diffusion equation with satellite absorption for three cases. (a) Radial diffusion with no pitch angle diffusion and no local source. (b) Radial and pitch angle diffusion but no local source. (c) Radial and pitch angle diffusion and a local source. The diffusion and source coefficients all have units of $\mathrm{s}^{-1}$. 
diffuse inward toward $L=7.5$, then remains constant inside $L=7.5$ where there are no losses and the eigenmodes do not decay.

A solution with radial diffusion, pitch angle diffusion and satellite sweeping is shown in Figure $12 b$. The steeper radial variation than the previous case is due to the finite lifetime of the lowest pitch angle eigenmode, and the shorter lifetimes of the higher order modes cause the large anisotropy near $L=7.5$ to decay beck to the lowest eigenmode by $L \approx 6$. The rapid pitch angle diffusion of electrons with $x<1$ near $L=7.5$ acts as a source for higher $x$ values leading to the local minima in $f$. However, there is a net loss of electrons with all pitch angles, so the deep local minima superimposed on a slowly increasing $f$ with $L$, evident in the results of the data analysis in the previous section, cannot be reproduced by this model.

Results of the model with an additional source of particles at all $L$ values are shown in Figure $12 c$. The source is represented by a negative lifetime, $-\tau_{5}$, so that the rapid change in pitch angle anisotropy near the minimum $L$, with a retum to the lowest eigenmode of pitch angle diffusion inside that $L$, is retained. In addition, the source provides deep local minima in $f$ and an outward displacement of the minima at larger $x$ resulting in a more isotropic distribution at the minimum $L$ itself.

A comparison of the solution in Figure $12 c$ with phase space densities derived from the data at constant $\zeta$ and $x$ is shown near the Ariel signature in Figure 13. Features in the data that are qualitatively reproduced by the model are the similar pitch angle distributions inside and outside the absorption region, the large anisotropy in the range $L=7.5$ to 8.5 , the isotropization (bunching of the contours) at high $x$ values just inside $L=7.5$, and the displacement of the phase space density minima to $L>7.5$ with increasing $x$. Note that when the high-order pitch angle eigenmodes are excited in the absorption region the distribution can no longer be well represented as $y^{2 n}$ as assumed in the data analysis. Therefore the phase space densities derived from the data in this region are expected to be valid over only a

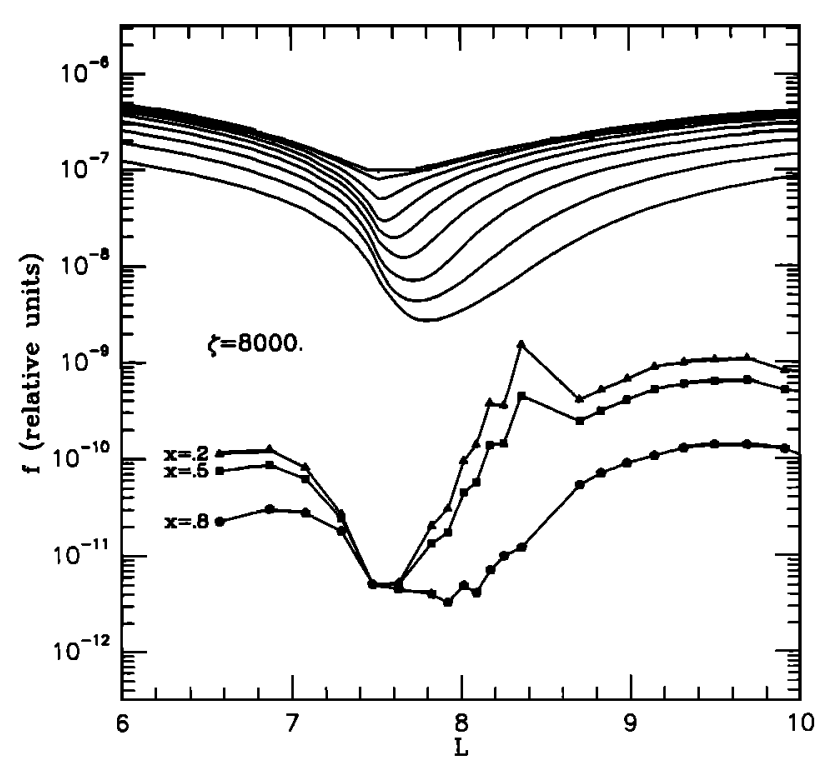

Fig. 13. A close-up view of the solution in Figure $12 c$ with the same format. The data points below are phase space densities derived from the data analysis at the indicated values of $x$ and $\zeta$ $\left(\mathrm{MeV} \mathrm{G}^{-1}\right)$. The vertical scales have been shifted for ease of comparison. limited range of $x$. The low $x$ values were not sampled near the Ariel minimum $L$ so that the model $f$ values at low $x$, which show only a shallow minimum, are not expected to be reproduced by the data in this region.

The necessity for a source term in the bimodal diffusion equation (14) has implications for the approximations that were made in its derivation. The first approximation was to describe radial diffusion assuming that $x$ and $\zeta$ are conserved rather than $M$ and $K$. Relaxing this assumption leads to additional diffusion coefficients, such as $D_{x \zeta}=\frac{\partial x}{\partial L} \frac{\partial \zeta}{\partial L} D_{L}$. These can be calculated and their equivalent source strength estimated by substituting the known electron distribution function in the relevant term of the diffusion equation. Such estimates lead to small equivalent sources relative to those required, indicating that the approximation is adequate for the observed electron distributions. The second approximation was to assume perfectly elastic pitch angle scattering. If some energy change is allowed then a $D_{\zeta \zeta}$ coefficient must be included, and if the changes in energy and pitch angle are correlated then the off-diagonal (cross diffusion) elements of the diffusion tensor (13) must be also. These are strong possibilities as they would be required in scattering by resonant wave particle interactions, where the electrons give energy to or receive energy from the wave. An estimate of the required degree of inelasticity can be made in this case.

For simplicity we assume that the pitch angle and energy change in each scattering event are uncorrelated so that no crossdiffusion terms are required. Then we assume that the average squared change in momentum $p$ is related to the average squared pitch angle change by $(\Delta p / p)^{2}=\delta^{2}(\Delta x)^{2}$, where $\delta$ is a constant. The energy diffusion coefficient is now $D_{\zeta \zeta}=4 \zeta^{2} \delta^{2} D_{x z}$ and the condition for nearly elastic scattering is $y \delta \ll 1$. The results in Figure $12 c$ show that $\tau_{\zeta}^{-1}=7 D_{x x}$ is required to approximate the Ariel signature. By substituting a power law energy spectrum into the $\zeta$ diffusion term of the diffusion equation, an equivalent lifetime is obtained which, in the relativistic limit, is $\tau_{\zeta}^{-1}=\delta^{2} D_{x x} \gamma(\gamma-3)$. This leads to a required $\delta \approx 0.6$ for the observed $\gamma \approx 6$. Therefore $y \delta<1$ for all $y$, but the condition of nearly elastic scattering is strictly satisfied only at small y (small pitch angles). Since pitch angle diffusion requires that each individual $\Delta x$ be small, the energy changes in each scattering event are also small relative to the total energy. These results show that energy diffusion may be a plausible source of energetic electrons, but a detailed calculation will require solutions of the full three-dimensional diffusion equation, including the crossdiffusion terms for resonant wave particle interactions. The observed hardening of the energy spectrum (decrease in $\gamma$ ) at $L \approx 7.5$ in Figure 5 , and softening immediately outside, may provide a test of such a model.

An order-of-magnitude estimate of the energy diffusion coefficient due to whistler mode turbulence has been given by Kennel [1969]. The equivalent coefficient for diffusion in speed $v$ is related to the pitch angle diffusion coefficient by $D_{w}=\left(V_{A}^{4} / v^{2}\right) D_{\text {axa }}$, where $V_{A}=B /(4 \pi \mathrm{mm})^{1 / 2}$ is the electron Alfven speed and $n$ is the local plasma number density. Converting to $D_{55}$ gives $\delta \approx\left(W_{A} / v\right)^{2}$ where $\gamma$ is now the relativistic Lorentz factor. A kinetic energy of $1 \mathrm{MeV}$ and the locally observed plasma density at $L=7.5$ inbound of $n \approx 0.06 \mathrm{~cm}^{-3}$ [Selesnick and $M c N u t t, 1987$ ] lead to $\delta \approx 5$. This is a larger energy diffusion coefficient than derived from the model described above, but given the level of approximation, the disagreement is not unreasonable. The determination of the plasma density at $L=7.5$ outbound was complicated by spacecraft charging, but may have 
been as high as $n \sim 1 \mathrm{~cm}^{-3}$ [McNutt et al., 1987] leading to $\delta \sim$ 0.3 .

The model described above is not flexible enough to accurately fit all of the phase space densities derived from the data. A simple way to include the sources and losses due to pitch angle diffusion is to solve the equation

$$
L^{2} \frac{\partial}{\partial L}\left[L^{-2} D_{L} \frac{\partial f}{\partial L}\right]=\frac{f}{\tau_{s s}}-\frac{f}{\tau_{m=t}}
$$

for each $K, M$ pair. Here the different metric from (14) is a result of using $K, M$ rather than $x, \zeta$ coordinates. The lifetime $\tau_{\text {net }}$ represents a net source which may include, in an approximate way, the losses due to pitch angle diffusion. The smooth curves in Figures 7 to 10 are from least-squares fits of parametrized solutions of (18) to the data points. The same numerical method as in the solution of (16) was used, absorption by all three satellites was included, and the data points at the highest and lowest $L$ values were used as boundary conditions. Four fit parameters for each curve, $D, \alpha, S, \beta$, describe the radial diffusion coefficient, $D_{L}=D(L / 7.5)^{\alpha}$, and the net source lifetime, $\tau_{=1}^{-1}=S(L / 7.5)^{\beta}$. The model parameters resulting from the fits are shown in Figure 14. Note the increase in $D$ and $S$ with $K$ required to fit the pitch angle distributions (except at $K=0.4$ where the model does not accurately fit the data). This is because of the larger range in $L$ of satellite absorption at higher $K$ values and is not required when pitch angle diffusion is included properly (as in the calculation of Figure 13).

The validity of the parameters should be judged by the quality of agreement between the solutions and data points in Figures 7 to

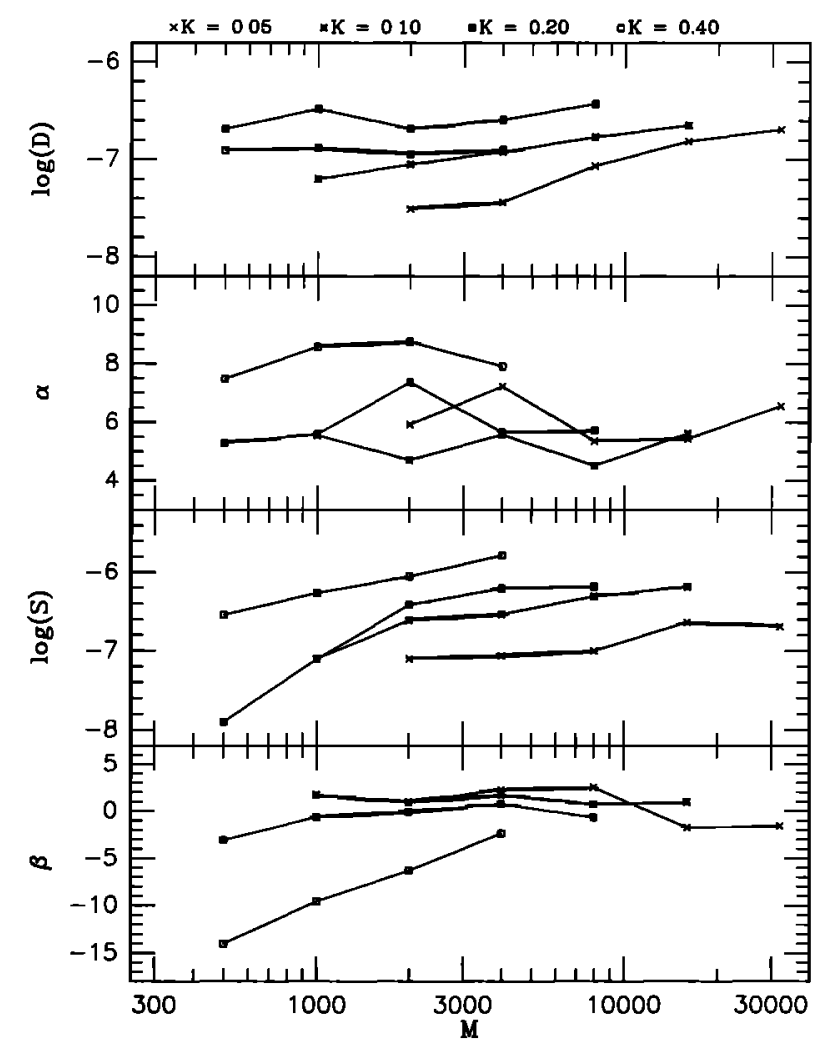

Fig. 14. Parameters derived for the model fits to the phase space densities in Figures 7 to 10 , as described in the text. The diffusion parameter $D$ and source parameter $S$ both have units of $\mathrm{s}^{-1}$.
10. Clearly, the solutions for $K=0.4 \mathrm{G}^{1 / 2} R_{U}$ do not reproduce the data accurately. The best fits are those for $K=0.1 \mathrm{G}^{1 / 2} R_{U}$, $M=4000$ and $8000 \mathrm{MeV} \mathrm{G}^{-1}$ which, according to Figure 6, are also the most accurately sampled by the CRS detectors. The parameters derived from these fits should be considered the most reliable. The radial diffusion coefficient at $L=7.5$ is $D$ $10^{-7} \mathrm{~s}^{-1}$ with $\alpha \sim 5$ to 7 and a net source strength $S-10^{-7} \mathrm{~s}^{-1}$. In general, the coefficients $D$ and $S$, and the exponent $\alpha$, are well constrained in the cases for which good fits were obtained, with typical uncertainties of $20 \%$ to $30 \%$. The exponent $\beta$ is less well constrained and the uncertainties are consistent with $\beta=0$. In many cases, the solutions fail to produce a local minimum in $f$ at Umbriel, indicating that the source strength near Umbriel was too small and the derived source strength applies to the Ariel signature. This may indicate that local increases in the source strength are required near the satellite absorption regions.

The radial diffusion coefficient estimated above from the CRS data can be compared with previous estimates from both CRS and LECP electron data. However, we must be careful to note that the value obtained above is dependent on other processes such as pitch angle and energy diffusion which may not be accurately modeled, so that only an order of magnitude agreement with previous estimates should be expected. Cooper and Stone [1991] estimate $D_{\boldsymbol{L}}-10^{-7}$ to $10^{-6} \mathrm{~s}^{-1}$ from the width of the CRS Ariel absorption signature. From a local analysis of the LECP phase space densities in the Ariel signature Cheng et al. [1987] find $D_{L}$ -5 to $9 \times 10^{-7} \mathrm{~s}^{-1}$. From a global analysis of the LECP phase space densities, similar to that based on (18), Hood [1989] finds $D_{L} \sim 6 \times 10^{-7} \mathrm{~s}^{-1}$ for $-30 \mathrm{keV}$ electrons at the Ariel signature. Thus there seems to be a general agreement in the approximate value of $D_{L}$ for electrons from tens of $\mathrm{keV}$ to $\mathrm{MeV}$ energies at $L \approx 7.5$. In addition, Hood [1989] finds a radial dependence of $D_{L L}$ with a power law exponent of 3 to 4 . This is somewhat lower than the values of $\alpha$ in Figure 14, but here the CRS results may be adversely influenced by the processes producing local minima in $f$ that are not present in the LECP data analyzed by Hood.

\section{CONCLUSION}

The radial profiles of the electron phase space density, $f$, derived for constant values of the first two adiabatic invariants, $M$ and $K$, are substantially different from expectations based on steady state radial diffusion and satellite absorption. First, there are deep local minima near the satellite minimum $L$, indicating an electron source for given $M$ and $K$ values, which may be due to the nonconservation of $M$ and/or $K$. We have used solutions of the radial diffusion equation to characterize the required source strength. The location in $L$ of the local minimum near Ariel is a function of both $K$ and $M$. For $K=0.1 G^{1 / 2} R_{U}$ and $M$ varying from 1000 to $8000 \mathrm{MeV} \mathrm{G}^{-1}$ the minimum varies from $L=7.6$ to 7.8. The Ariel minimum $L$ is $7.5 \pm 0.1$. For higher $K$ values it is an increasing function of $K$, reaching $L \approx 8.2$, independent of $M$, at $K=0.4 G^{12} R_{U}$. The strong $K$ dependence is due to the different locations of the inbound and outbound counting rate minima (Figure 4). The Umbriel minima do not appear to be significantly displaced from the minimum $L$ of about 10.4 (although there is some indication of a small outward displacement at the higher $K$ and $M$ values), even though the inbound (low $K$ ) counting rate data do show a clear minimum at $L=10.7$ (see Figure 4 or Cooper and Stone [1991]). The differing displacements of the Ariel and Umbriel signatures may be a result of the higher $K$ values sampled near the Ariel 
minimum $L$ (Figure 6), so that a high $K$ displacement in the Umbriel signature would not be well represented in the data. The counting rate data also show outward displacements of the Miranda signature at high $K$, but the phase space density analysis cannot be extended to this region with the CRS data. The displacement of the Ariel signature can be reproduced in the solutions of the diffusion equation if a sufficiently strong source is included, and in that case its dependence on $K$ is a result of the similar dependence of the absorption efficiency. These models imply that the existence of local minima in $f$ is necessary to produce the offsets which were observed directly in the raw data, thereby supporting the conclusion that a local electron source is required.

The nearly uniform electron pitch angle anisotropy, except for sharp variations in the satellite signatures, is also inconsistent with the expectations of steady state radial diffusion. This has led to the conclusion that pitch angle diffusion must be important in order to return the distribution function to the lowest pitch angle eigenmode away from the satellite signatures. Inelastic pitch angle scattering can also provide a natural source of electrons to produce the local minima in $f$ by causing energy diffusion along the steep gradient in the electron energy spectra. Observations by the Voyager 2 LECP instrument at energies just below the threshold of the CRS detectors used in this study are consistent with the results presented here and show that the local minima in $f$ are present only for $M \geq 100 \mathrm{MeV} \mathrm{G}^{-1}$ [Cheng et al., 1987]. In the energy diffusion interpretation, this would be consistent with the hardening of the electron spectra in the LECP energy range [Mauk et al., 1987].

Observations of intense whistler mode waves by the plasma wave instrument in the vicinity of the Ariel signature provide a direct estimate of local pitch angle diffusion coefficients. Coroniti et al. [1987] show that for two $L$ values, 6.73 and 7.64, electrons in an energy range of -5 to $50 \mathrm{keV}$ are expected to be on strong pitch angle diffusion with diffusion coefficients in the range of $\sim 10^{-4}$ to $10^{-3} \mathrm{~s}^{-1}$. At higher energies, electrons are not on strong diffusion, but the coefficients extrapolated to $\sim 1 \mathrm{MeV}$ are still near $-10^{-4} \mathrm{~s}^{-1}$, considerably higher than the value of $-10^{-8} \mathrm{~s}^{-1}$ that is sufficient in the model we have presented. It may be that the low value applies away from the Ariel region. Larger wave amplitudes are expected in the vicinity of the satellite absorption, where the highly anisotropic electron distributions may be unstable to whistler growth. The reason that only a small value of the pitch angle diffusion coefficient is required to produce the observed pitch angle distributions is that the derived radial diffusion coefficients are also small, so that the high-order pitch angle eigenmodes that are excited in the absorption regions have time to decay while the particles have diffused only a short radial distance. Localized enhancements of pitch angle diffusion in the absorption regions may increase the possibility that the source required to explain the phase space density minima is a result of inelastic pitch angle scattering.

The large pitch angle diffusion coefficients estimated from the wave data would lead to fast precipitation losses of radiation belt particles and require a similarly strong source to replenish them. This is true for particles at energies where no extrapolation of the estimated diffusion coefficients is required as well as at the CRS energies. Fast radial diffusion could replenish the precipitation losses but would be incompatible with the radial diffusion coefficients derived from the particle data. Coroniti et al. [1987] suggest that this problem can be overcome by a source due to whistler mode energy diffusion. However, any source sufficient to replenish losses at the rate suggested by the wave data would dominate the losses by satellite absorption. The wave intensities therefore still appear to be inconsistent with the existence of absorption features in the particle data. In fach the radial and pitch angle diffusion coefficients, or equivalent source strengths, estimated in the present work are near the upper limits of those compatible with the observed particle absorption features.

The existence of definite particle sink due to satellite absorption, with strong variations in the sweeping rate as a function of $L$ and equatorial pitch angle, produces distinctive features in the particle distribution function. These have led to conclusions about diffusion in the radiation belt of Uranus which would not be available in the absence of the satellites.

\section{APPENDIX}

\section{Detector Response Functions}

An electron telescope identical to that on the Voyager CRS instrument was irradiated with a wide, monoenergetic electron beam and the distribution of pulse heights in the detector of interest was recorded. With the known incident electron flux and experimental livetime, the response function, $R\left(E, E^{\prime}=E_{b}, \theta=\theta_{b}\right)$, was calculated for the particular beam energy, $E_{b}$, and direction, $\theta_{b}$, which were varied to provide a complete characterization of the detector response.

The results of a DI calibration with $E_{b}=2.36 \mathrm{MeV}$ and $\theta_{b}=0$ (along the telescope axis) are shown in Figure Al, which is a plot of $R$ versus $E$. The response function shows a sharp, full energy peak at $E \approx 2 \mathrm{MeV}$ due to electrons which stop in D1 losing most of their energy to ionization within the detector (the remainder is lost to ionization within the thin aluminum casing on the front of the telescope, and the thermal blanket which surrounds the instrument). The broad peak centered at $E \approx 1 \mathrm{MeV}$ is due to electrons which penetrate the D1 detector. There is a low background level (evident below $E=0.8 \mathrm{MeV}$ ) due to backscattering and scattering through the sides of the detector. The lack of response below $E=0.5 \mathrm{MeV}$ is due to the electronic threshold.

The entire D1 calibration, integrated over $\theta$, is displayed in Figure A2. $R$ is plotted versus both $E$ and $E^{\prime}$. We have used a smoothing procedure in the $E$ direction and a cubic $s$ line

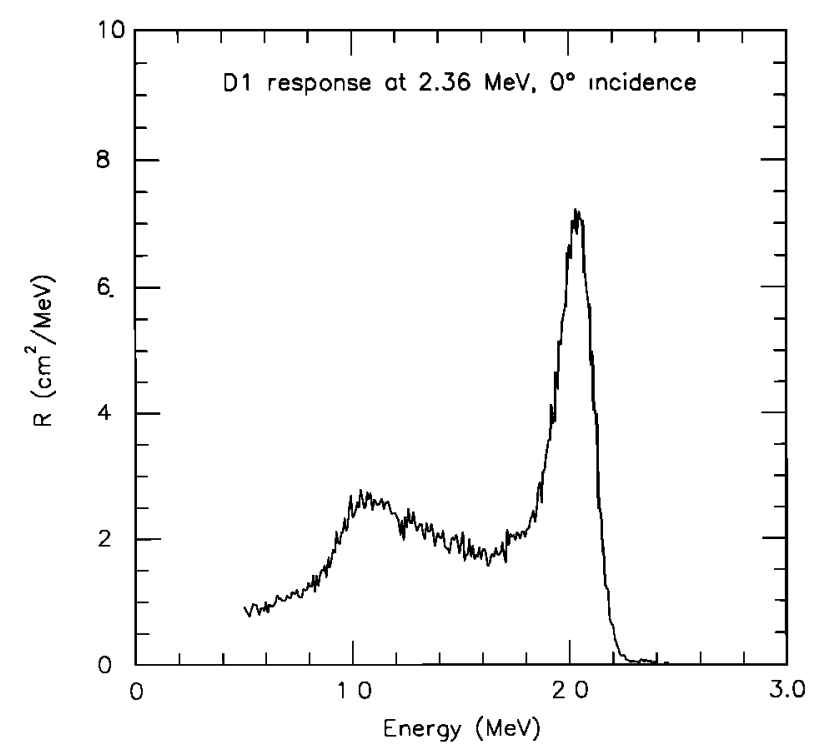

Fig. A1. Measured D1 response function for $2.36 \mathrm{MeV}$ electrons entering along the TET axis. 


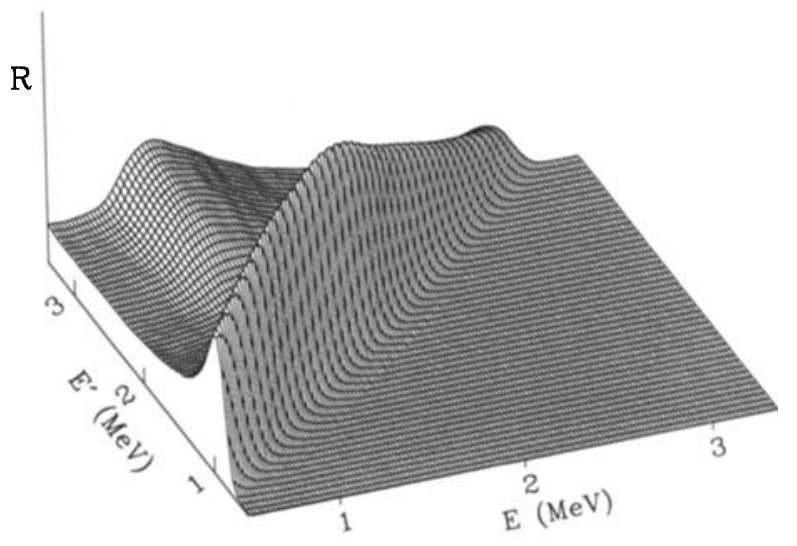

Fig. A2. The D1 response function versus incident energy, $E^{\prime}$, and observed energy, $E$.

interpolation in the $E^{\prime}$ direction to obtain the resolution shown. Clearly evident is the variation of the two peaks seen in Figure Al. The stopping peak is dominant at low energies, where no electrons have sufficient energy to penetrate D1. At higher energies the penetrating peak becomes dominant.

To characterize the properties of D1 and D2 as omnidirectional integral flux detectors, Figure $A 3$ shows the two response functions integrated over $\theta_{b}$ and observed energy, $E$. The $y$ axis is labeled $A \Omega$ because it represents an energy dependent geometry factor. That is, $A \Omega\left(E^{\prime}\right) j\left(E^{\prime}\right) d E^{\prime}$ is the counting rate for electrons in the energy range between $E^{\prime}$ and $E^{\prime}+d E^{\prime}$. We see from the slope of the curves at low energies that the detectors do not have a well-defined energy threshold. Instead, their sensitivity is an increasing function of the electron energy. The D2 geometry factor is lower than that of D1 primarily due to the shielding of D2 by D1.

\section{Deadtime Correction}

After an event is recorded by the electronic components of CRS, there is a short deadtime during which subsequent events

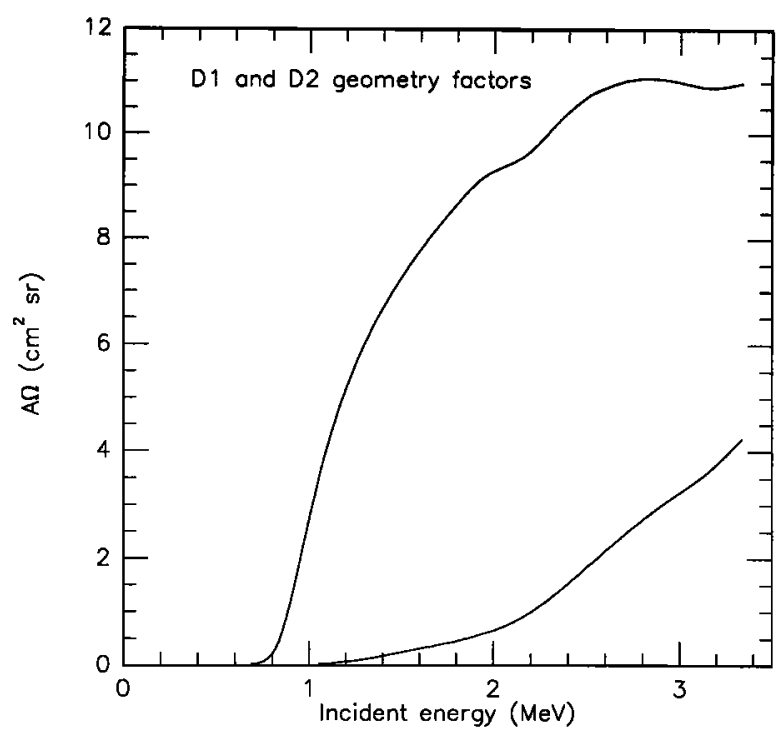

Fig. A3. D1 (top curve) and D2 geometry factors versus electron kinetic energy. are not recorded. The measured counting rates are thèrefore lower than they would be for an ideal instrument; an effect which increases with the counting rate. Due to the high electron fluxes encountered at Uranus, it is necessary to correct for the deadime effect. An observed rate, $r^{\text {obs }}$ (counts per second), is related to the true rate (that which would be observed if the deadtime were zero), $r^{\text {tro }}$, by $\left(r^{\text {obo }}\right)^{-1}=\left(r^{\text {tro }}\right)^{-1}+\tau^{d}$ where $\tau^{d}$ is the deadtime. Then

$$
r^{\infty}=\frac{r^{\text {tron }}}{1+\tau^{2} r^{\text {trose }}}
$$

Using identical electronics to those aboard Voyager, we have measured the deadtime applicable to the TET data. It is a function of energies of both the event under consideration and the previous event. Therefore we obtain a deadtime matrix $\tau_{i j}^{d}$ where $i$ labels the PHA channel number with the energy of the event and $j$ labels that of the previous event. By requiring that (A1) hold for integral rates with constant deadtime, its generalization becomes

$$
r_{i}^{\text {obs }}=\frac{r_{i}^{\text {true }}}{1+\sum_{j} \tau_{i j}^{d} r_{j}^{\text {trese }}}
$$

where $r_{i}^{\text {truodoby) }}$ is the true (observed) rate in channel $i$. The average deadtime for the data we have analyzed is about $20 \mu \mathrm{s}$.

\section{Pileup Correction}

Another effect which occurs at high counting rates is pulse pileup, whereby two or more events which occur within the amplifier pulse width add and become a single event at higher amplitude. The correction of true rates for pulse pileup is difficult, but fortunately it is usually small. We have developed an analytic approach which we have checked by Monte Carlo simulations. When the correction is small the analytic results are acceptable. We approximate the pulse shape by a square pulse of width $\tau=2 \mu \mathrm{s}$ (the amplifier shaping time). The probability of no events occurring in channel $j$ during the time $\tau$ is $e^{-\tau_{j}}$, where $r_{j}$ is the rate of such events. Then the rate of events in channel $i$ which follow events in channel $j$ within time $\tau$, but with no other events in any other channel during this time, is $r_{i}\left(1-e^{-r_{j}}\right) e^{-\tau R}$, where $R$ is the integral rate. The pileup correction to the rate in channel $k$ has a contribution from events which would be in lower channels but pile up into $k$, and a negative contribution from events which would be in channel $k$ but pile up to higher channels. Including both contributions, the rate corrected for pileup of two events only is

$$
\begin{aligned}
r_{k}^{P 2}=r_{k} & +e^{-\tau R}\left[\sum_{i=1}^{k-1} r_{i}\left(1-e^{-\nabla_{k-i}}\right)\right. \\
& \left.-\sum_{i=1}^{N}\left[r_{i}\left(1-e^{-\tau_{k}}\right)+r_{k}\left(1-e^{-\nabla_{i}}\right)\right]\right]
\end{aligned}
$$

where the superscript $\boldsymbol{P} 2$ refers to the two-event pileup corrected rate, and $N$ is the total number of channels. The rate of two-event pileups can be combined in a similar way with the singles rate to obtain the three-event pileup correction, and so on. Only twoevent pileup has been used in the data analysis.

\section{Satellite Sweeping Lifetime}

The satellite sweeping lifetime, $\tau_{s s}$, is the inverse probability of absorption per unit time averaged over many satellite orbits. In 
a dipole magnetic field, neglecting the satellite orbital speed relative to the electron drift speed in the corotating frame, it is related to the satellite orbital period $T_{s}$, the electron angular drift speed $\omega_{d}$, and the satellite $L$ shell contact time $\Delta t$, by [Peonessa and Cheng, 1987]

$$
\tau_{s s}=\frac{\pi T_{s}}{\omega_{d}\left(\Delta t^{+}+\Delta t^{-}\right)}
$$

For a dipole offset along the planetary rotation axis by a distance $z_{0}$ there are two contact times per satellite orbit [Pconessa and Cheng, 1987; Cooper, 1990]. For $L \geq 1.01 L_{\min }$ :

$$
\Delta t^{ \pm}=\frac{T_{S}}{2 \pi A}\left[\frac{\Delta L L_{\min }}{L^{2}\left[1-L_{\min } L\right]^{1 / 2}\left|\sin \phi_{\text {atb }}^{ \pm}\right|}\right]
$$

for $L_{\min }-\Delta L \leq L \leq L_{\min }+\Delta L$ :

$$
\Delta I^{ \pm}=\frac{T_{S}}{2 \pi A}\left[\frac{L-L_{\min }+\Delta L}{L+\Delta L}\right]^{1 / 2}
$$

and for $L_{\min }+\Delta L<L<1.01 L_{\min }$ :

$$
\Delta t^{ \pm}=\frac{T_{S}}{2 \pi A}\left[\left[\frac{L-L_{\min }+\Delta L}{L+\Delta L}\right]^{1 / 2}-\left[\frac{L-L_{\min }-\Delta L}{L-\Delta L}\right]^{1 / 2}\right]
$$

where

$$
\begin{gathered}
\cos \phi_{\text {otb }}^{ \pm}=\frac{-B \pm\left[1-\frac{L_{\min }}{L}\right]^{1 / 2}}{A} \\
A=\frac{a \sin \eta}{\left(a^{2}+z_{0}^{2}\right)^{1 / 2}} \quad B=\frac{z_{0} \cos \eta}{\left(a^{2}+z_{0}^{2}\right)^{1 / 2}}
\end{gathered}
$$

$\eta$ is the tilt angle of the dipole axis from the planetary rotation axis, $a$ is the satellite orbital radius, and $L_{\min }$ is the orbital minimum $L$ of the satellite. Notice that $T_{S}$ does not enter the final expression for $\tau_{s s}$. The range of $L$ covered by the satellite radius at a given time, $\Delta L$, increases with magnetic latitude $\lambda$ and for a dipole field, assuming that the satellite is small relative to the scale size of the gradient of $L$, is given by

$$
\Delta L=\frac{\left(r_{m a t}+r_{g}\right)}{R_{U}} \frac{\left(1+4 \tan ^{2} \lambda\right)^{1 / 2}}{\cos ^{2} \lambda}
$$

where $r_{\mathrm{an}}$ is the satellite radius and $r_{\mathrm{g}}$ is the electron gyroradius. For Uranus $\eta=60^{\circ}, z_{0}=0.31 R_{U}$, and $r_{\text {mat }}=242 \mathrm{~km}$ for Miranda, $580 \mathrm{~km}$ for Ariel, and $595 \mathrm{~km}$ for Umbriel. For $\omega_{d}$ in a dipole field we use the approximation given by Schulz and Lanzerotti [1974]. Finally, following Cooper [1990], we neglect the corkscrew and leapfrog effects due to the finite electron gyroradius.

Ackoowledgments. Several people at Caltech have made significant contributions to the CRS calibrations and we thank especially M. Looper, A. Curmmings, J. Cooper, D. Burke, M. Tran and K. Hargreaves. We also thank B. Mauk for providing electron data from the LECP experiment ( $S$. M. Krimigis, principal investigator). This work was supported by NASA under contract NAS7-918 and grant NGR 05-002-160.

The editor thanks J. F. Cooper and L. L. Hood for their assistance in evaluating this paper.

\section{REFERENCES}

Acuna, M. H., J. E. P. Connemey, and N. F. Ness, Implications of the GSFC Q3 model for trapped particle motion, J. Geophys. Res., 93, 5505-5512, 1988.
Baker, S. and R. D. Cousins, Clarification of the use of chi-square and likelihood functions in fits to histograms, Nucl. Instrwm. Methods Phys. Res., 221, 437-442, 1984.

Cheng, A. F., S. M. Krimigis, B. H. Mauk, E. P. Keath, C. G. Maclennan, L. J. Lanzerotti, M. T. Paonessa, and T. P. Amstrong, Energetic ion and electron phase space densities in the magnetosphere of Uranus, $J$. Geophys. Res., 92, 15,315-15,328, 1987.

Connemey, J. E. P., M. H. Acuna, and N. F. Ness, The magnetic field of Uranus, J. Geophys. Res., 92, 15,329-15,336, 1987.

Cooper, J. F., Satellite sweeping of electrons at Neptune and Uranus, Geophys. Res. Lett., 17, 1665-1668, 1990.

Cooper, J. F. and E. C. Stone, Electron signatures of satellite sweeping in the magnetosphere of Uranus, J. Geophys. Res., in press, 1991.

Cooper, J. F. and E. C. Stone, Satellite signatures from MeV electrons in the magnetosphere of Uranus (abstract), Eos Trans. AGU, 67, 1174, 1986.

Coroniti, F. V., W. S. Kurth, F. L. Scarf, S. M. Krimigis, C. F. Kennel, and D. A. Gumett, Whistler mode emissions in the Uranian radiation belts, J. Geophys. Res., 92, 15,234-15,248, 1987.

Haerendel, G., Diffusion theory of trapped particles and the observed proton distribution, in Earth's Particles and Fields, edited by B. M. McComac, pp. 171-191, Reinhold, New York, 1968.

Hood, L. L., Radial diffusion in the Uranian radiation belts: Inferences from satellite absorption loss models, J. Geophys. Res., 94, $15,077-15,088,1989$.

Kennel, C. F., Consequences of a magnetospheric plasma, Rev. Geophys., $7,379-419,1969$.

Krimigis, S. M., T. P. Armstrong, W. I. Axford, A. F. Cheng, G. Gloeckler, D. C. Hamilton, E. P. Keath, L. J. Lanzerotti, and B. H. Mauk, The magnetosphere of Uranus: Hot plasma and radiation environment, Science, 233, 97-102, 1986.

Mauk, B. H., S. M. Krimigis, E. P. Keath, A. F. Cheng, T. P. Armstrong, L. J. Lanzerotti, G. Gloeckler, and D. C. Hamilton, The hot plasma and radiation environment of the Uranian magnetosphere, J. Geophys. Res., 92, 15,283-15,308, 1987.

Mcllwain, C. E., Magnetic coordinates, Space Sci. Rev., 5, 585-598, 1966.

McNutt, R. L. Jr., R. S. Selesnick, and J. D. Richardson, Low-energy plasma observations in the magnetosphere of Uranus, J. Geophys. Res., $92,4399-4410,1987$.

Ness, N. F., M. H. Acuna, K. W. Behannon, L. F. Burlaga, J. E. P. Connemey, R. P. Lepping, and F. M. Neubauer, Magnetic fields at Uranus, Science, 233, 85-89, 1986.

Paonessa, M. T. and A. F. Cheng, Satellite sweeping in offset, tilted dipole fields, J. Geophys. Res., 92, 1160-1166, 1987.

Press, W. H., B. P. Flannery, S. A. Teukolsky, and W. T. Vetterling, Numerical Recipes: The Art of Scientific Computing, Cambridge University Press, New York, 1986.

Roederer, J. G., Dynamics of Geomagnetically Trapped Radiation, Springer, New York, 1970.

Schulz, M. and L. J. Lanzeroti, Particle Diffision in the Radiation Belts, Springer, New York, 1974.

Selesnick, R. S. and R. L. McNutt, Jr., Voyager 2 plasma ion observations in the magnetosphere of Uranus, J. Geophys. Res., 92, 15,249-15,262, 1987.

Stone, E. C., The physical significance and application of $L, B O$, and $R O$ to geomagnetically trapped particles, J. Geophys. Res., 68, 4157-4166, 1963.

Stone, E. C., J. F. Cooper, A. C. Cummings, F. B. McDonald, J. H. Trainer, N. Lal, R. McGuire, and D. L. Chenette, Energetic charged particles in the Uranian magnetosphere, Science, 233, 93-97, 1986.

Stone, E. C., R. E. Vogh, F. B. McDonald, B. J. Teegarden, J. H. Trainer, J. R. Jokipii, and W. R. Webber, Cosmic ray investigation for the Voyager missions: Energetic particle studies in the outer heliosphere - and beyond, Space Sci. Rev., 21, 355-376, 1977.

Tarantola, A. and B. Valette, Generalized nonlinear inverse problems solved using least squares criterion, Rev. Geophys., 20, 219-232, 1982.

Walt, M., Radial diffusion of trapped particles, in Particles and Fields in the Magnetosphere, edited by B. M. McCormac, Pp. 410-415, D. Reidel, Hingham, Mass., 1970.

R. S. Selesnick and E. C. Stone, 220-47 Califomia Institute of Technology, Pasadenn, CA 91125.

(Received September 6, 1990;

revised November 5, 1990;

accepted December 6, 1990.) 